\title{
CONOCIMIENTOS SOBRE PLANTAS RITUALES UTILIZADAS POR YERBATERAS DE LOS MERCADOS DE QUITO, ECUADOR: APORTES SOBRE SU ESTADO DE CONSERVACIÓN
}

\author{
KNOWLEDGE ABOUT RITUAL PLANTS USED BY HERBALIST FROM THE MARKETS \\ OF QUITO, ECUADOR: CONTRIBUTIONS ON THEIR CONSERVATION STATUS
}

\author{
Marco A. RODRÍGUEZ-SEGOVIA*, Camila RUBIO-JIMÉNEZ, Katherine NARVÁEZ-VERDESOTO \\ y Jhon TUZ-CHAMORRO
}

Universidad Central del Ecuador, Facultad de Ciencias Biológicas y Ambientales. E-mail: *marcozeus24@gmail.com

Submitted: 09/06/2020; Accepted: 02/08/2020

\section{RESUMEN}

En Ecuador existen 5.172 especies de plantas útiles, el $20 \%$ son usadas en los llamados usos sociales que incluyen rituales, creencias, mitos y prácticas religiosas. Nosotros consideramos todo esto dentro de una única categoría, la categoría ritual. El propósito de esta investigación fue la de describir y recopilar el propósito de uso ritual, relacionado a varias creencias en plantas utilizadas por yerbateras en mercados de Quito, sin olvidarnos del lado biológico-científico que ello implica. Además de responder a varias preguntas. Buscamos yerbateros en los mercados: Central, San Roque, Cotocollao, Santa Clara y San Francisco. Entre estos lugares entrevistamos a 32 yerbateros. Nos basamos en la observación directa y en el método de entrevista-compra. Se determinó el índice de valor de uso de una especie por varios informantes (IVUs) y el Índice de Friedman (IF). Revisamos el estado de conservación y distribución de las especies. Encontramos 90 especies de plantas rituales repartidas en 43 familias botánicas. Entre las familias más usadas destacan Asteraceae (12\%) y Lamiaceae (11\%). Entre las plantas con mayor aceptación cultural estaban Ruta graveolens y Ambrosia arborescens. Un 82\% de las plantas provenían de la Sierra ecuatoriana, 9\% de la Costa y 8\% de la Amazonía. Un $40 \%$ de las plantas encontradas eran introducidas, un 59\% eran nativas, una especie era endémica. Dos especies están extintas en estado silvestre (EW) y una está en peligro de extinción (EN). Los conocimientos de las yerbateras no aumentan de forma significativa con la edad y experiencia $(\mathrm{p}>0.05)$ como tampoco son diferentes entre mercados $(\mathrm{p}>0.05)$. En conclusión, basados en los propósitos de uso recopilados se describen usos rituales nuevos para varias especies. Las especies de origen europeo introducidas tienen un fuerte arraigo en prácticas rituales, mientras muchas especies nativas son poco conocidas y su uso es menos destacable.

Palabras-clave: Baños energéticos; Limpias; Mercados; Ritual; Yerbateras

\begin{abstract}
In Ecuador there are 5.172 useful plant species, $20 \%$ are used in so-called social uses that include rituals, beliefs, myths and religious practices. We consider all this within a single category, the ritual category. The reason of this investigation was to describe and compile the purpose of the ritual use, related to various beliefs in plants used by herbalists in Quito markets, without forgetting the biological-scientific side that this implies. We also respond to some questions. We looked for yerbateros in the markets: Central, San Roque, Cotocollao, Santa Clara and San Francisco. In these places we interviewed 32 yerbateros, based on direct observation and the interview-purchase method. We calculated two indices : The Use Value (For one species across all informants) (UVs) and the Informant consensus of Friedman (IF). We reviewed the conservation and distribution status of the species. We found 90 species of ritual plants in 43 botanical families. Asteraceae (12\%) and Lamiaceae (11\%) stand out among the most used families. Among the plants with the greatest cultural acceptance there were Ruta graveolens and Ambrosia arborescens. 82\% of the plants came from the Ecuadorian Sierra, 9\% from the Coast and $8 \%$ from the Amazon. $40 \%$ of the plants found were introduced, $59 \%$ were native, one specie was endemic. Two species are extinct in the wild (EW) and one specie is endangered (EN). The herbalist knowledge does not increase significantly with age and experience $(\mathrm{p}>0.05)$, nor are they different between markets $(\mathrm{p}>0.05)$. In conclusion, based on the collected purposes of use, new ritual uses for various species are described. The introduced species of european origin have a strong influence in ritual practices, while many native species are little known and their use is less remarkable.
\end{abstract}

Keywords: Energetic Baths; Limpias; Markets; Ritual; Herbalist 


\section{INTRODUCCIÓN}

Ecuador posee una gran diversidad florística, en el país se han registrado más de 17.000 especies de plantas, de las cuales 4.500 especies son endémicas (VALENCIA et al., 2000; MAE, 2010; LEÓNYÁNEZ et al., 2011). De acuerdo a la Enciclopedia de plantas útiles del Ecuador, de las 5.172 especies de plantas útiles en el país, apenas el $20 \%$ de estas son utilizadas en los llamados usos de tipo social (DE LA TORRE et al., 2008). Estas son plantas asociadas a creencias y mitos, con caracteres religiosos o místicos y que pueden utilizarse incluso para curar malestares físicos o del alma. Los usos sociales de las plantas se desglosan en las categorías de tipo ritualístico/religiosas, estimulantes, vomitivos, materiales para fumar drogas, supresoras del apetito y agentes de infertilidad. Estas plantas tienen un fuerte arraigo en las costumbres de las personas, involucrando muchas perspectivas para su uso. En este trabajo consideraremos este tipo de prácticas junto a sus procesos de preparación como "ritual" y a las plantas usadas en ello como "plantas rituales".

En Ecuador el uso de plantas rituales típicamente se ha documentado en las 14 etnias del país, que son principalmente comunidades rurales y de las cuales destacan en su mayoría las comunidades de la Sierra y Amazonía (DE LA TORRE et al., 2008; CABANILLA, 2015; CLAVIJO y YANEZ, 2017). En estas comunidades, quiénes llevan a cabo prácticas rituales son los denominados: curanderos, hechiceros, chamanes o Yachags (BECERRA et al., 2020), los cuales utilizan las plantas para propósitos como evocar fuerza física, alejar malas energías, realizar curaciones, atraer suerte, contactar con espíritus, etc. (KVIST y MORAES, 2006). Así, los estudios etnobotánicos han permitido rescatar información sobre los usos tradicionales de este tipo de plantas (DE LA TORRE et al., 2008; CARAPIA y VIDAL, 2013).

Los mercados tradicionales son una fuente inexplorada para las investigaciones etnobotánicas, en ellos se puede adquirir información sobre la biodiversidad, el manejo y el universo cultural de las poblaciones en estudio (MAIOLI-AZEVEDO y DA FONSECA-KRUEL, 2007). Para Díaz et al. (2011), los mercados son un reflejo de cultura ya que en ellos se da la satisfacción de muchas de las necesidades físicas (alimento, vestido, salud, etc.), sociales, económicas y culturales de los pobladores de una región. En Quito los mercados y ferias públicos son de vital importancia para el abastecimiento de alimentos, ya que abastecen a cerca del 75\% de la población (HOLLENSTEIN, 2019).

Mientras que la mayoría de estudios etnobotánicos realizados en mercados suelen abarcar varias categorías de uso para las plantas (ZAMBRANO-INTRIAGO et al., 2015), pocos son los estudios con un enfoque más profundo de lo que se puede razonar como ritual (KVIST y MORAES, 2006; MAIOLI-AZEVEDO y DA FONSECA-KRUEL, 2007). Muchos de esos estudios se han enfocado en plantas medicinales (MAIOLI-AZEVEDO y DA FONSECA-KRUEL, 2007), posiblemente debido a su potencial terapeútico y económico, con una potencial ventaja para las industrias farmacéuticas (RAMOS et al., 2007; MAIOLI-AZEVEDO y DA FONSECA-KRUEL, 2007).

Dentro de los mercados, los yerbateros representan perfectamente las relaciones existentes entre el ser humano y el reino vegetal (BARRERA y KUKLINSKI, 2018). Un yerbatero es una persona cuyo oficio se trata de colectar y vender plantas con varios usos, principalmente medicinales (ÁLVAREZ y MIRANDA, 2014; BARRERA y KUKLINSKI, 2018), aunque también con propósitos alimenticios, rituales, ornamentales, etc. Los conocimientos de los yerbateros son pasados de forma oral en cada generación (RIOS et al., 2007; ÁLVAREZ y MIRANDA, 2014; BARRERA y KUKLINSKI, 2018).

En países como Brasil se les denomina "erveiros" (del portugués erveiro=hierba), cuya traducción es hierbero (LOPES et al., 2010). En Colombia como yerbateros, curanderos e hechiceros (BARRERA y KUKLINSKI, 2018). En México y según Díaz et al. (2011) como comerciantes de plantas. En inglés se les conoce como "herbalist", que significa naturópata o herbolario (ADAMS et al., 2007); otra traducción es la de "herb seller" o vendedor de hierbas (MAIOLI-AZEVEDO y DA FONSECAKRUEL, 2007). Sin embargo, el contexto y percepciones sobre lo que es un yerbatero al parecer son distintos en otros lugares de América.

En Brasil y el Chocó de Colombia los yerbateros tienen una fuerte influencia africana y muchos de ellos cultivan sus propios productos (LOPES et al., 2010; BARRERA y KUKLINSKI, 2018). En Brasil un buen número de ellos son tanto mujeres como varones (MAIOLI-AZEVEDO y DA FONSECA-KRUEL, 2007; LOPES et al., 2010). En comparación, los yerbateros de la ciudad de Quito en la región Sierra del Ecuador, son en su mayoría mujeres mestizas muy apreciadas por la comunidad (Figura 1) debido a los amplios conocimientos que dominan sobre determinadas especies de plantas, en especial sus propiedades y sus formas de uso (ÁLVAREZ y MIRANDA, 2014; 
BARRERA y KUKLINSKI, 2018). No cultivan sus propios productos y parecen tener una fuerte influencia de la colonización española.

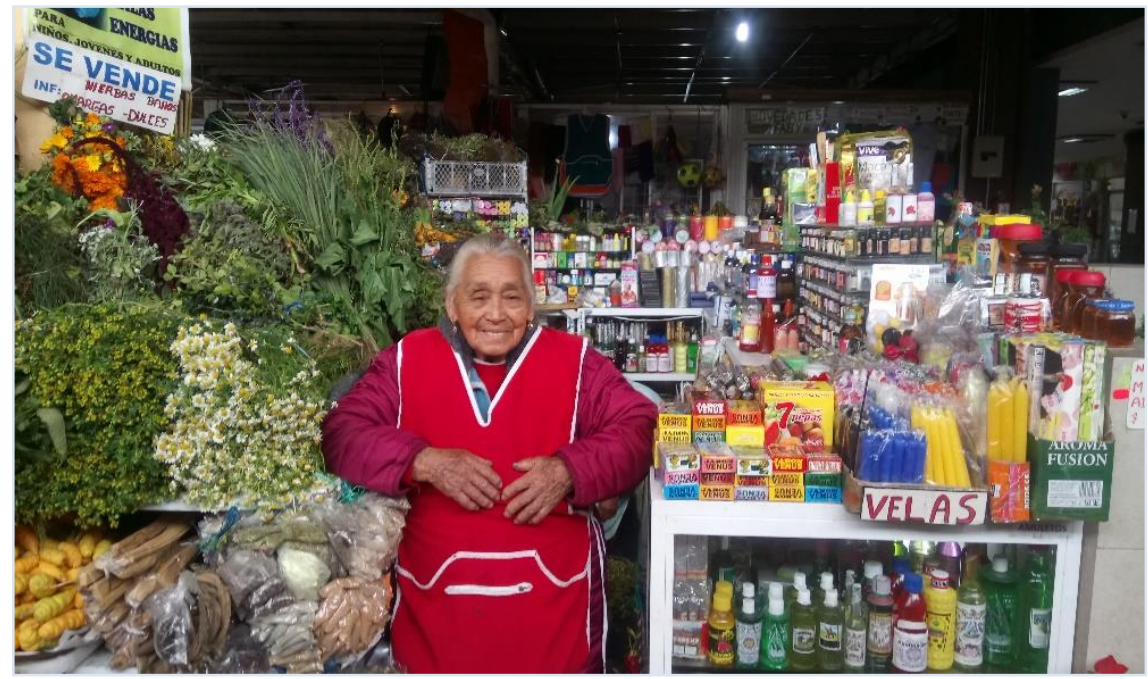

Figura 1: Olga Chiluisa, yerbatera del mercado de Cotocollao, Quito-Ecuador.

Las yerbateras de Quito utilizan un delantal para evitar ensuciarse, algunas lo usan de color blanco para representar pureza. Muchas colocan su nombre en su puesto de venta con su número de teléfono. En sus puestos de venta, además de plantas, ofrecen velas, inciensos y un sinnúmero de brebajes y preparaciones a base de colonias con plantas para la realización de hechizos. Algo similar ocurre con los hombres que tienen esta profesión.

En Quito las yerbateras también son llamadas curanderas y una parte de ellas se dedican a ser parteras que brindan a asesoría a mujeres gestantes (ÁLVAREZ y MIRANDA, 2014). Las yerbateras tienen la capacidad de sanar diversos males en las personas por medio de las denominadas "limpias" (ÁLVAREZ y MIRANDA, 2014). Una limpia es una práctica que involucra un proceso de preparación de varias especies de plantas para propósitos como alejar malas energías, espíritus malvados o demonios y de alguna forma sanar a las personas de algún malestar (ÁLVAREZ y MIRANDA, 2014). Existen muchas prácticas rituales poco conocidas sobre estas yerbateras que vale la pena documentar.

Muchas especies de plantas utilizadas por yerbateros no son cultivadas, una buena parte evidentemente son tomadas directamente de la naturaleza. El impacto que pueda generar este hecho en la conservación de las especies silvestres se desconoce por completo. La mayoría de las 3.508 (78\%) especies endémicas del Ecuador están amenazadas en algún grado; de estas 2.080 (46\%) se consideran vulnerables (VU), $1.071(24 \%)$ en peligro (EN) y 353 (8\%) en peligro crítico (CR) (LEÓN-YÁNEZ et al., 2011). Es preocupante no disponer de información acerca de la abundancia, distribución, tasas y formas de extracción de poblaciones naturales de plantas en los trópicos; por estos motivos es importante realizar estudios etnobotánicos para evitar la pérdida de saberes tradicionales, conservando la herencia cultural y la biodiversidad (JARAMILLO et al., 2014).

El propósito de esta investigación fue la de describir y recopilar el propósito de uso ritual, relacionado a varias creencias en plantas utilizadas por yerbateras en mercados de Quito, sin olvidarnos del lado biológico-científico que ello implica. También se buscó responder algunas preguntas: ¿De acuerdo a índices etnobotánicos cuáles son las plantas rituales de mayor importancia cultural y cuáles son las más efectivas para resolver problemas en creencias similares tales como alejar el mal de aire o malas energías, alejar malos espíritus, curar el espanto, quitar el estrés, quitar la brujería, etc.? ¿Son diferentes los conocimientos y las especies de plantas encontrados entre cada mercado?

Además, y de manera secundaria utilizamos datos obtenidos por casualidad en este trabajo para responder la pregunta: ¿Existe alguna relación significativa entre la edad de las yerbateras con sus conocimientos y tiempo de experiencia? Realizamos este trabajo hasta el inicio de la Pandemia por SARS-Cov-2. 


\section{MATERIAL Y MÉTODOSO \\ Área de Estudio}

En el Distrito Metropolitano de Quito existen unos 42 mercados y ferias libres temporales (HOLLENSTEIN, 2019). Seleccionamos cinco mercados de la ciudad donde existen yerbateros con amplios conocimientos sobre plantas. Estos mercados fueron el Mercado Central, El Mercado de San Roque, El Mercado de Cotocollao, El Mercado Santa Clara y el Mercado San Francisco (Figura 2). Estos mercados fueron elegidos de manera preferencial por ser de los más concurridos en Quito. Reciben productos agropecuarios, piscícolas, textiles, entre otros, de las tres regiones del Ecuador: Costa, Sierra y Amazonía. Son abastecidos por una gran cantidad de intermediarios y productores independientes. Además, son muy antiguos.
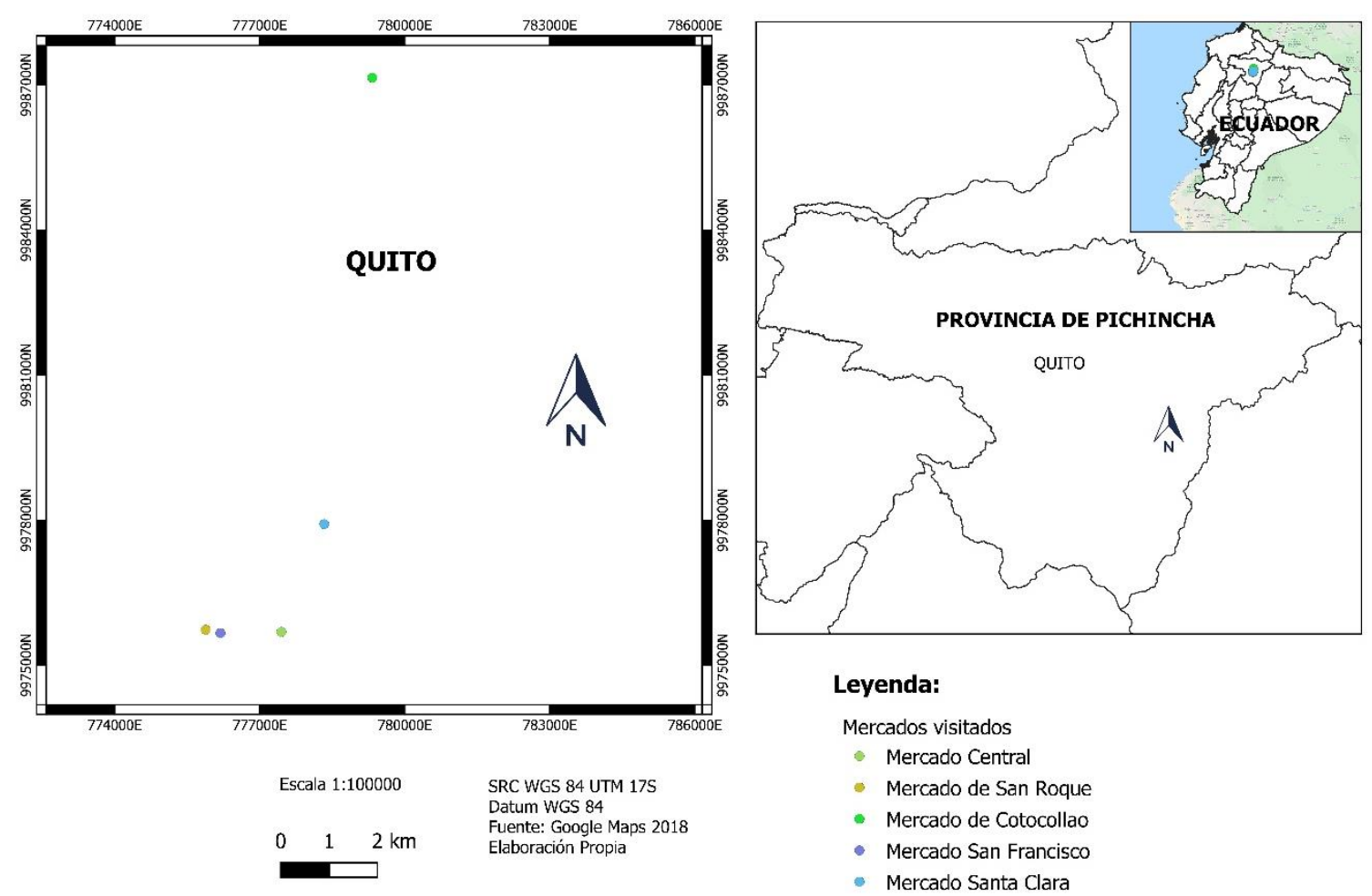

Figura 2: Ubicación de los diferentes mercados en la ciudad de Quito.

\section{Entrevistas con yerbateros de varios mercados}

La mayoría de estudios etnobiológicos recomiendan una muestra de al menos 35 entrevistas o superior (HOFFMAN y GALLAHER, 2007; RODRÍGUEZ et al., 2018). En esta ocasión nuestro esfuerzo de muestreo fueron 32 entrevistas realizadas a yerbateros en los mercados mencionados anteriormente (Tabla 1).

Nuestro criterio de selección para las entrevistas consistió en trabajar con cualquier yerbatero que se dedicara a la venta de plantas de origen nativo o introducido y de uso local (MAIOLI-AZEVEDO y DA FONSECA-KRUEL, 2007; DÍAZ et al., 2011). Realizamos un sondeo piloto previo, como primer acercamiento, donde buscamos la presencia de yerbateros que tenían puestos fijos dentro y alrededor de los mercados. También se incluyeron a yerbateros con puestos semi-establecidos en los alrededores de los mercados (muchos de ellos frecuentan un lugar en específico donde se instalan para trabajar) (DÍAZ et al., 2011). Los yerbateros que aceptaron ser entrevistados lo hicieron bajo su consentimiento previo informado, todos fueron informados de los objetivos de esta investigación, de modo que no se necesitó de un comité de ética.

Empleamos entrevistas con preguntas abiertas y el método de entrevista-compra que se establece con los yerbateros (MAIOLI-AZEVEDO y DA FONSECA-KRUEL, 2007; DÍAZ et al., 2011). Las entrevistas tuvieron dos rondas. En la primera ronda de entrevistas se incluyeron preguntas que tenían que ver con conocimientos sobre plantas rituales, sus propósitos y formas de uso, su procedencia, otros usos que pudiesen tener (los otros usos mencionados por los yerbateros se discutirán al final de todos los resultados) (Ver Anexo 2). Durante las entrevistas algunas yerbateras realizaron demostraciones de 
cómo utilizaban sus plantas en rituales y nos proporcionaron un poco de sus conocimientos al respecto, recogimos estos aspectos haciendo hincapié sobre en el uso ritual. En la segunda ronda solo se visitaron los mercados para corroborar y corregir la información provista con cada yerbatero en la primera ronda. Esto entre los meses de Noviembre y Diciembre del 2019. La edad de los yerbateros estaba entre 20 a 85 años con un promedio de edad de 54 años. Se entrevistaron a 5 hombres y 27 mujeres.

Tabla 1: Número de yerbateros entrevistados en cada mercado visitado. Nota: el número de yerbateros observados es un aproximado. El secretismo de muchos yerbateros evitó que aceptasen participar y nosotros respetamos eso.

\begin{tabular}{lcc}
\hline \multicolumn{1}{c}{ Mercados visitados } & Número de yerbateros observados & $\begin{array}{c}\text { Número de yerbateros que accedieron a } \\
\text { participar con nosotros }\end{array}$ \\
\hline Mercado de Cotocollao y lugares & 5 & 4 \\
aledaños & 7 & 2 \\
Mercado Santa Clara & 5 & 4 \\
Mercado Central & 17 & 15 \\
Mercado San Roque & 10 & 7 \\
Mercado San Francisco y lugares & 44 & 32 \\
aledaños & 4 & \\
Total & & \\
\hline
\end{tabular}

\section{Identificación taxonómica}

Utilizamos la guía ilustrada de Quintana (2013), la observación directa y conocimiento previo de la flora nativa para la identificación taxonómica de las plantas encontradas. Aunque la mayoría de las plantas fueron bien identificadas por los yerbateros, nuestro trabajo consistió en comprar, a manera de colecta botánica, a las especies de plantas que pudimos y especialmente a las que no pudimos reconocer en la forma en la que son vendidas (la planta completa o parte de ellas, e inclusive atados de plantas), verificamos taxonómicamente algunas plantas en el Herbario Alfredo Paredes (QAP) de la Universidad Central del Ecuador (UCE). Esto con la ayuda de literatura especializada y del experto botánico Carlos Cerón Martínez. Algunas plantas fueron identificadas solo hasta el nivel taxonómico de género y no fueron depositadas en el herbario debido al cierre de sus operaciones durante la actual Pandemia por SARS-Cov-2.

\section{El estado de conservación y distribución de las especies encontradas}

Para revisar el estado de conservación y distribución de las plantas, utilizamos el libro rojo de las plantas endémicas del Ecuador de León-Yánez et al. (2011), el check list digital de la lista roja de la UICN (2020) y literatura al respecto. Por conveniencia los datos tomados en las entrevistas sobre la procedencia de las plantas se discuten seguidos de estos aspectos.

\section{Índices}

Para responder a la primera pregunta planteada en la introducción utilizamos el índice de valor de uso de una especie por varios informantes de Phillips y Gentry (1993a, 1993b), que permite conocer la importancia cultural de una especie (IVUs $=\Sigma \mathrm{iVUis} / \mathrm{Ns}$, donde: VUis $=$ Valor de uso de una especie por cada informante y Ns= Número de informantes entrevistados para la especie s) y el índice de Friedman et al. (1986), que permite estimar la importancia relativa de cada especie a partir del grado de consenso de los informantes $(\mathrm{IF}=(\mathrm{Ip} / \mathrm{It}) \times 100$, donde: Ip: número de informantes que mencionaron una especie (frecuencia de mención); It: número total de informantes) (apud HOFFMAN y GALLAHER, 2007). Todos los cálculos se realizaron en base a los 32 yerbateros que accedieron a participar con nosotros.

\section{Procesamiento de la información y análisis de datos}

Todos los datos fueron sistematizados en respuestas generales dentro de una base de datos, algunos fueron organizados de acuerdo a la frecuencia de ocurrencia con las que fueron citadas por los yerbateros. El número de conocimientos se midió de acorde al número de especies citadas por cada yerbatera. Para responder nuestra segunda y tercera preguntas de la introducción utilizamos las 
pruebas estadísticas de Kruskall Wallis y una correlación de Pearson usando el programa BioEstat 5.3. Usamos Pearson por su robustez estadística frente a desviaciones de normalidad (MCDONALD 2014). Con ayuda del programa R-Studio visualizamos si las plantas en cada mercado son diferentes mediante un escalado multidimensional no métrico (NMDS). La matriz base se construyó colocando a las especies de plantas mencionadas en filas y en columnas a los yerbateros de cada mercado, registrando cada dato obtenido como especie mencionada (1) o no mencionada (0), siguiendo de forma parecida a Jaramillo et al. (2014).

\section{RESULTADOS Y DISCUSIÓN}

\section{Familias botánicas más usadas por yerbateras de Quito}

Entre todos los lugares visitados encontramos un total de 90 especies diferentes de plantas que tienen un uso ritual, estas especies estaban repartidas en 43 familias botánicas. Las familias más usadas por los yerbateros son Asteraceae (12\%), Lamiaceae (11\%), Solanaceae (8\%) y Fabaceae (6\%) (Tabla 2). De la Torre et al. (2008) mencionan que de hecho Lamiaceae es una de las familias con más registros para usos rituales, la característica de olores fuertes y atractivos presentes en las especies de esta familia al parecer las hace plantas sujetas a muchas creencias. Solanaceae es otra familia con bastantes registros de usos rituales según De la Torre et al. (2008). Nuestro estudio además destaca un buen número de registros para familias como Asteraceae y Fabaceae.

Tabla 2: Familias más usadas por los yerbateros. En la tabla se agruparon a 28 familias que tienen puntuaciones individuales de un $1.11 \%$.

\begin{tabular}{lc}
\hline Familia & Frecuencia de ocurrencia \\
\hline Asteraceae & $12 \%$ \\
Lamiaceae & $11 \%$ \\
Solanaceae & $8 \%$ \\
Fabaceae & $6 \%$ \\
Myrtaceae & $4 \%$ \\
Rosaceae & $4 \%$ \\
Piperaceae & $3 \%$ \\
Poaceae & $3 \%$ \\
Verbenaceae & $3 \%$ \\
Boraginaceae & $2 \%$ \\
Burseraceae & $2 \%$ \\
Lauraceae & $2 \%$ \\
Melastomataceae & $2 \%$ \\
Urticaceae & $2 \%$ \\
Xanthorrhoeaceae & $2 \%$ \\
Otras 28 familias & $31 \%$ \\
\hline
\end{tabular}

\section{Entrevistas con yerbateras de varios mercados}

Plantas utilizadas por las yerbateras para realizar una limpia: escobas amargas y dulces

Para ejecutar una limpia se necesitaba la preparación de una o la combinación de varias especies de plantas en un atado (varias plantas atadas con una cuerda). Las yerbateras preparaban los atados para venderlos o para que estas los usen con las personas que pagan por hacerse una limpia dentro de los mercados. Existen dos tipos de atados acorde al conocimiento tradicional de las yerbateras: el primero, conocido como "escoba amarga", es elaborado con plantas amargas de olor fuerte no muy agradable y se usa para realizar limpias a los adultos; el segundo, conocido como "escoba dulce", es elaborado con plantas de olor agradable y dulce y se usa para realizar limpias a niños y jóvenes (Tabla 3). 
Tabla 3: Especies utilizadas para elaborar escobas amargas y dulces según las yerbateras de varios mercados de Quito. Nota: los atados de escoba amarga y escoba dulce pueden variar de acuerdo a la disponibilidad de ciertas plantas.

\begin{tabular}{ll}
\hline Tipo de escoba & \multicolumn{1}{c}{ Especies utilizadas } \\
\hline Escoba amarga & Buddleja bullata, Varronia sp. Ruta graveolens, Ambrosia arborescens, Urtica leptophylla, \\
& Urtica dioica, Miconia crocea, Tagetes erecta, Cupressus lusitánica, Piper aduncum, Cestrum \\
& affine, Dodonaea viscosa, Tanacethun partenium, Psidium guajaba, Juglans neotrópica, Tagetes \\
& minuta, Pinus patula, Eucaliptus globulus, Prunus serotina, Tecoma stans, Margyricarpus \\
& pinnatus, Croton ferrugineus, Annona cherimola, Inga insignis, Morella pubescens, Brugmansia \\
& arbórea, Monnina phillyreoides. \\
\hline Escoba dulce & Rosmarinus officinalis, Lavandula angustifolia, Persea americana, Eriobotrya japónica, \\
& Corimbya citriodora, Psidium guajava, Myrcianthes hallii, Mentha pulegium, Matricaria \\
& chamomilla, Cymbopogon citratus, Pelargonium citrosum \\
\hline
\end{tabular}

Según las yerbateras, las personas acudían con más frecuencia a consultar sus conocimientos y solicitar sus servicios para realizar limpias u otras prácticas rituales en Navidad y fin de año. En otros países como México suele existir una mayor afluencia a los vendedores de plantas en determinadas épocas del año, donde se celebran tradiciones relacionadas a prácticas religiosas, ceremoniales y rituales; en estas épocas se venden algunas especies en específico (DÍAZ et al., 2011).

\section{La limpia como práctica ritual}

Una limpia se ejecuta de forma tradicional por el yerbatero, para esto el yerbatero toma el atado de plantas y golpetea con este alrededor del cuerpo de una persona (Figura 3), mientras tanto rocía un poco de colonia con un atomizador pequeño además de una bebida alcohólica (trapiche, aguardiente o cualquier otra bebida alcohólica) que es escupida con los labios a manera de rocío.

\section{Baños Rituales}

Las escobas amargas y dulces son también utilizados para los denominados "baños energéticos" (baños que mejoran la energía de una persona), en los que se usa la decocción de las plantas, estos podían ser respectivamente "baños amargos" o "baños dulces" (Anexo 1). Algunos yerbateros simplemente mencionaron especies utilizadas en baños energéticos o simplemente en baños, sin clasificarlas como dulces o amargos. Maioli-Azevedo y Da Fonseca-Kruel (2007) se refieren a este tipo de prácticas como baños ritualísticos, sin clasificarlos como amargos y dulces.

Otros baños rituales utilizaban una única especie de planta y no un atado. Especies como la "Arrastradora" (Selaginella diffusa) son utilizadas en baños para atraer el amor, el "Abre caminos" (Kohleria sp.) era utilizada para realizar baños de buena suerte, la "Destrancadera" (Monochaetum lineatum) se usaba para realizar baños que atraigan clientes a los negocios, la "Amanzaguapos" (Justicia sp.) se utiliza en baños con el propósito de dominar a una persona con mal genio y enamorarla; siendo recomendada para mujeres que buscan la obediencia de sus parejas. Aquí es necesario señalar que los baños con fines amorosos podían ser para atraer posibles parejas o servir en los denominados "amarres amorosos", en los que se busca atraer el amor de una persona en específico.

Este estudió logró documentar por primera vez el propósito de uso ritual de algunas plantas, por ejemplo, de Selaginella diffusa. Cerón (2003) mencionó el uso de la planta en la categoría de uso mítico, pero no su propósito, de manera similar ocurre dentro de La Enciclopedia de plantas útiles del Ecuador de De la Torre et al. (2008) donde solo es mencionada su especie. En esta misma Enciclopedia no figura Monochaetum lineatum. Por ende, el uso ritual que encontramos para esta especie es nuevo. Esto aplica para muchas de las especies observadas. 


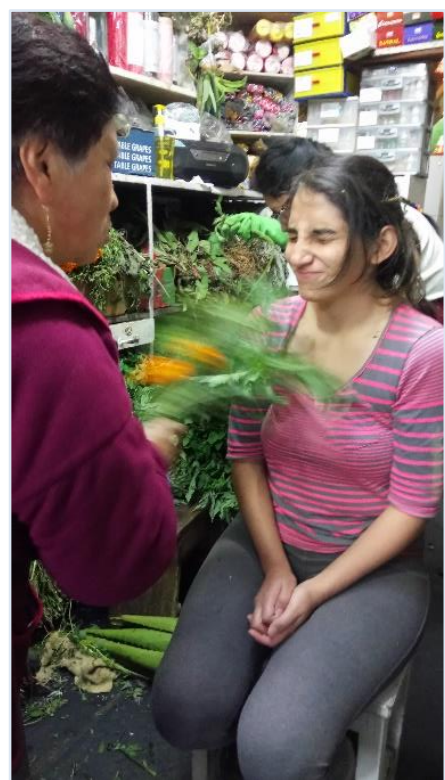

Figura 3: Katherine Narváez-Verdesoto siendo bofeteada con un atado de plantas durante una limpia. Curiosamente la yerbatera en la fotografía, además de otros entrevistados, mencionó tener la capacidad de presentir cuando una persona tenía malas energías o estrés mientras realizaban una limpia.

Plantas como Ruta graveolens, Cymbopogon citratus, Mentha pulegium, Rosmarinus officinalis, Artemisia absinthium, Tagetes erecta, Bixa Orellana, Ocimum basilicum y Zingiber officinale., también han sido reportadas como rituales por estudios de Maioli-Azevedo y Da Fonseca-Kruel (2007); los autores estudiaron estas plantas desde un enfoque medicinal y ritualístico.

\section{Parte de la planta utilizada}

Por orden de ocurrencia de entre las 90 especies identificadas, las hojas son usadas en un $86 \%$, los tallos en un $77 \%$, las flores en un $28 \%$, los frutos en un $3 \%$ y las semillas en un $1 \%$. De algunas especies solo se usaba una parte en exclusivo, así en el $13 \%$ de las especies solo se usan las hojas, en un $4 \%$ solo se usan los tallos, en un $2 \%$ las cortezas, en un $7 \%$ se usan las flores y en un $1 \%$ se usan los frutos. Consideramos que la planta se usaba por completo cuando se recomendaba su uso al menos en combinación de las hojas y tallos al mismo tiempo, de este modo en un $72 \%$ de las especies se usaba la planta completa.

En estudios de Maioli-Azevedo y Da Fonseca-Fruel, (2007) junto a Zambrano-intriago et al. (2015) se consideró importante señalar qué partes de las plantas son usadas por una comunidad para destacar aspectos culturales de uso. Desde la perspectiva medicinal que tuvieron estos trabajos es común que las partes más utilizadas de las plantas sean las hojas debido a que su actividad metabólica tiene propiedades farmacéuticas (JARAMILLO et al., 2014). En nuestros resultados ocurre algo similar sin tener mucha consideración por el lado medicinal.

Algunas prácticas realizadas con determinadas partes de las plantas llamaron nuestra atención. Las yerbateras elaboraban un collar con hilo rojo y 12 frutos de Tzímbalo (Solanum caripense) para colocarlo en el cuello de los niños. Supuestamente si un niño tenía "Mal de ojo" los frutos del collar se volverían de color negro (Figura 4). Las flores del Chamico (Datura satramonium) y el Guanto (Brugmansia sanguinea) se usaban únicamente para baños. En ocasiones, plantas como Ruta graveolens y Aloe vera son simplemente cultivadas en macetas porque al parecer su presencia aleja malas energías. De hecho, A. vera suele colgarse en la entrada de las casas con este propósito (Anexo $1)$.

\section{Distribución y Estado de Conservación}

Un 40\% (36) de las plantas encontradas eran introducidas, un 59\% (53) eran nativas y un 1\% (1) era endémica; esta última era la especie Phytelephas aequatorialis (Anexo 1). Encontramos que un $38 \%$ (34) de las especies están en la categoría de preocupación menor (LC), de las cuales 56\% (19) son introducidas. Un 57\% (51) de las especies están no evaluadas (NE), de las cuales el 69\% (35) son 
nativas. Olea europea está en la categoría de datos insuficientes (DD). P. aequatorialis está casi amenazada (NT), Juglans neotropica se encuentra en peligro de extinción (EN). Existen dos especies que están extintas en estado silvestre (EW), pero que son cultivadas, estas especies eran: Brugmansia sanguínea y Brugmansia arborea. Especies como Spartium junceum y Urtica leptophylla fueron colocados en la categoría LC en base a informes realizados por CONABIO (2016) junto a Hanan y Mondragón (2009) y Rodríguez et al. (2014), respectivamente. Otros estudios etnobotánicos como los de Díaz et al. (2011) reportaron la presencia de 17 especies de plantas protegidas por la ley de México en mercados de Tuxtla de Gutiérrez.

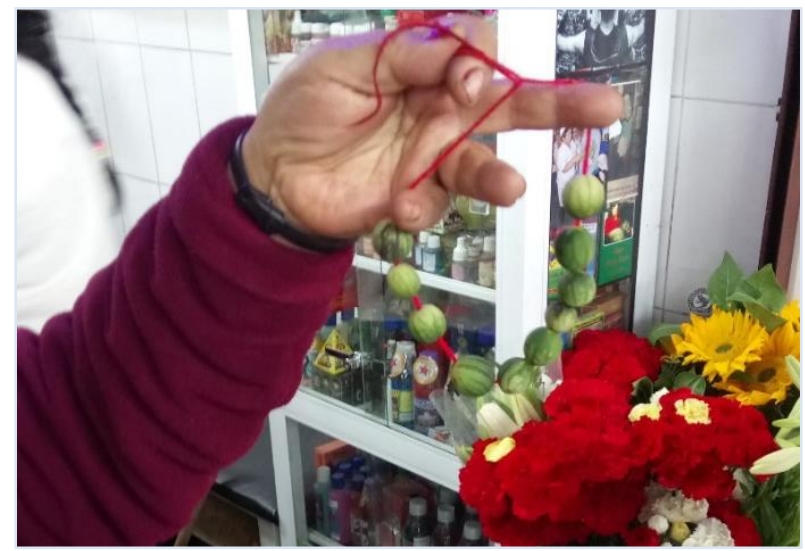

Figura 4: Un collar de frutos de Solanum caripense.

Muchas de las plantas encontradas son nativas, poco conocidas y se desconoce el estado de conservación puesto que no ha sido evaluado. Del mismo modo se desconoce la frecuencia de uso y biomasa traída a los mercados. Especies como Selaginella diffusa, por características de su biología reproductiva y requerimientos del hábitat, hace que sea difícil pensar en que fuese cultivada, es evidente que esta especie es tomada directamente de la naturaleza y desconocemos el impacto generado en sus poblaciones. De esta especie hallamos una muy pequeña cantidad en los mercados. En este sentido consideramos que el estatus de conservación biológica no solo contribuye a emprender acciones para preservas especies, también ayuda a reflexionar sobre la conservación de conocimientos tradicionales. Después de todo, los usos rituales de una especie también pueden estar en peligro.

Las plantas introducidas europeas tienen un cultivo muy extendido en Ecuador y algunas se han naturalizado en los ecosistemas, adaptándose a una variedad de hábitats y volviéndose especies invasoras (MAE, 2010). Varias de estas especies fueron colocadas como no evaluadas, al no hallar información en las fuentes revisadas sobre evaluaciones de sus poblaciones naturales. Para MaioliAzevedo y Da Fonseca-Kruel (2007) las plantas europeas introducidas tienen una fuerte influencia respecto del uso ritual. Estas plantas se han vuelto muy comunes culturalmente. Hay que señalar que procesos de transculturalización pueden provocar que las personas o grupos humanos adquieran elementos culturales prestados de otra cultura, en nuestro caso plantas introducidas (FARIÑA et al., 2011). Con el tiempo estos procesos terminan por generar aculturación, un estado en el que básicamente una cultura ha incorporado elementos de una cultura ajena, sustituyendo su cultura original. (GARCÍA y FIGUEROA, 2007; FARIÑA et al., 2011; ZEBADÚA, 2011). Desde nuestra perspectiva este es uno de los factores por el que este tipo de plantas fueron algunas de las más citadas por las yerbateras y que tuvieran una gran aceptación cultural de acuerdo a los índices que se analizarán más adelante.

\section{Procedencia de las plantas}

La gran mayoría de los yerbateros supieron respondernos únicamente con las localidades de las que sus proveedores traían las plantas, desconocían si eran plantas cultivadas o tomadas de la naturaleza. Una yerbatera mencionó que Cestrum affine y Miconia crocea son plantas que crecen en montes y que es común tomarlas de allí, dos yerbateras mencionaron la procedencia de especies silvestres de páramos del país como: Clinopodiun nubigenium, Lasiocephalus ovatus, y Chuquiraga jussieui.

Las localidades mencionadas por los yerbateros son muy variadas, la gran mayoría provienen de Ambato (22\%) y Latacunga (22\%) (Figuras 5 y 6). La mayoría de lugares mencionados pertenecen a 
la región Sierra del Ecuador y representan en conjunto un $82 \%$ de la procedencia de las plantas hacia los mercados visitados. Algunos yerbateros mencionaron la procedencia de plantas de la región Costa (9\%) y Amazonía (8\%), pero no sabían de una localidad más exacta. Varios yerbateros del Mercado Central y el Mercado San Francisco mencionaron que el Mercado de San Roque en la ciudad de Quito era su fuente de abastecimiento.

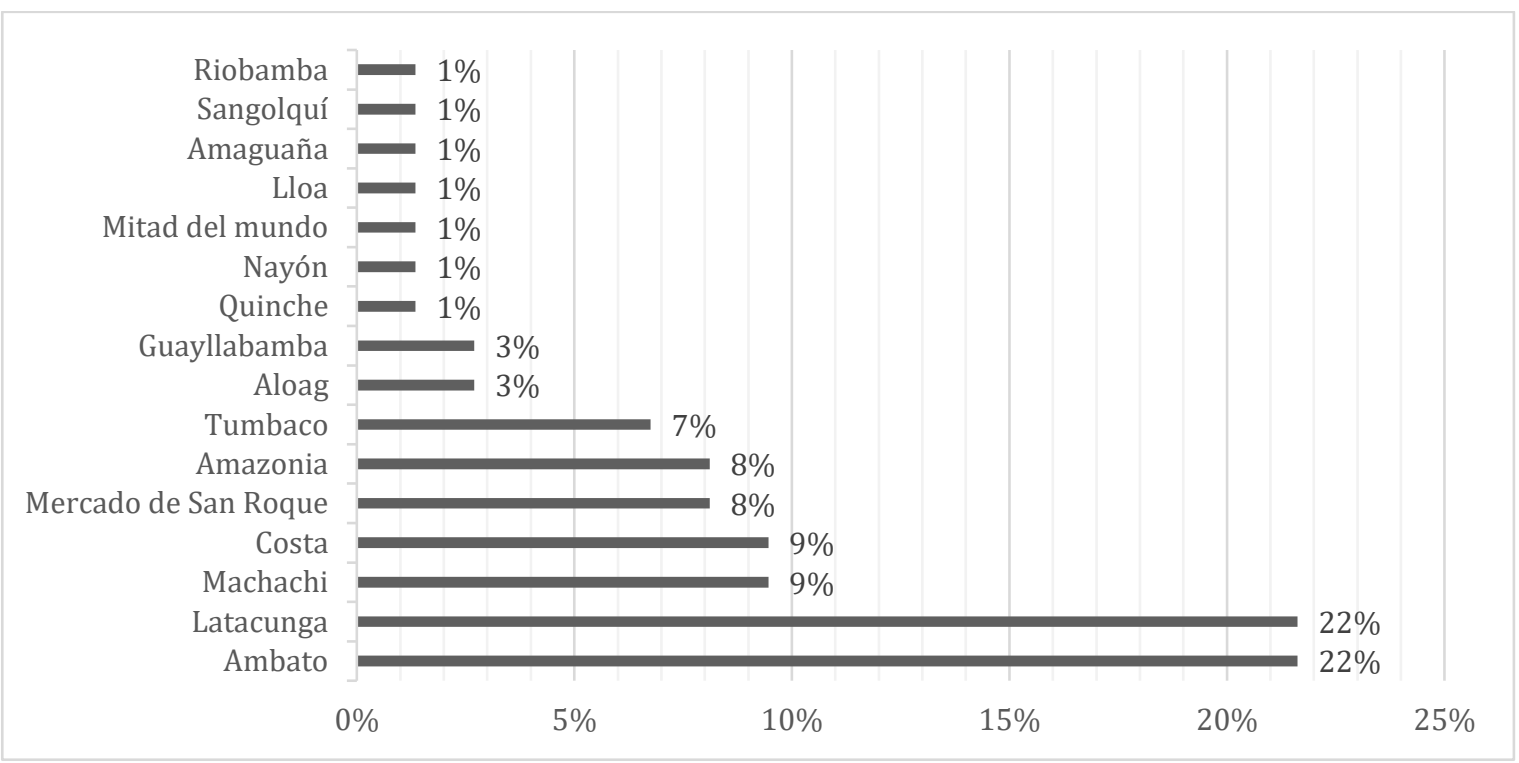

Figura 5: Procedencia de las plantas entre los mercados visitados de acuerdo a su frecuencia de ocurrencia.

Sería recomendable una investigación más a fondo con los proveedores de las yerbateras para saber el verdadero origen de las plantas que traen. Un buen ejemplo es el estudio etnobotánico de MaioliAzevedo y Da Fonseca-Kruel (2007) en ferias libres de las zonas Norte y Sur de Rio de Janeiro donde reportan que un $20 \%$ de las plantas provienen del extractivismo predatorio, mientras que según Azevedo y Silva (2006) en la zona oeste de Río de Janeiro fue de un $48.8 \%$ de las especies medicinales y rituales. Otros estudios similares en comunidades campesinas encuentran que la mayoría de las plantas son tomadas directamente del campo en los alrededores de una comunidad (JARAMILLO et al., 2014). Considere que el extractivismo predatorio puede ser una gran amenaza para la conservación de las especies y del patrimonio cultural.

\section{Importancia cultural de acuerdo a los índices IVUS e IF}

De acuerdo al IVUs, la especie con mayor aceptación cultural entre los yerbateros fue Ruta graveolens (1,28), seguida de Ambrosia arborescens (1), Urtica urens (0,97), Baccharis latifolia $(0,91)$, Tanacetum parthenium $(0,84)$ y Cestrum affine $(0,78)$ (Anexo 1$)$. Altos valores de este índice indican que las especies son usadas para diversos propósitos mientras que valores pequeños son para usos más específicos (MARÍN-CORBA et al., 2005). Este índice realiza una valoración cultural adecuada en entornos culturales determinados; de manera que mientras una especie se use en varios ámbitos tendrá mayor importancia para un grupo humano (JARAMILLO et al., 2014). Consideramos que fue adecuado en ámbitos rituales.

Según el IF, un 90,63\% de los yerbateros estarían de acuerdo en que $R$. graveolens es la planta más efectiva para usos rituales relacionados a creencias similares como: alejar el mal de aire o malas energías, alejar malos espíritus, curar el espanto, quitar el estrés, quitar la brujería, etc. Para pocas especies este consenso marcado por el IF fue bastante elevado, siendo mayor al $70 \%$, mientras que para la gran mayoría de las especies no superaba el 3,13\%. Un bajo consenso en el IF se podría considerar síntoma de transculturización, donde existe pérdida de los conocimientos tradicionales de las plantas, aunque también podría tratarse de un deterioro en la transmisión intergeneracional de conocimientos e inclusive de la rareza de las especies (JARAMILLO et al., 2014).

$R$. graveolens es una planta bastante mencionada en estudios etnobotánicos (MAIOLI-AZEVEDO y DA FONSECA-KRUEL, 2007; ZAMBRANO-INTRIAGO et al., 2015). $R$. graveolens es la segunda de las plantas con mayor número de registros medicinales en la Enciclopedia de plantas útiles del 
Ecuador, mientras que A. arborescens es la séptima especie de planta con mayor número de registros entre varias categorías de uso (Alimenticio, Alimento de invertebrados, Apícola, Materiales, Medioambiental, Social y Tóxico) para el país (DE LA TORRE et al., 2008). El resto de las especies con índices altos encontrados, pertenecen a familias que son muy importantes para suplir algunas necesidades medicinales y rituales en comunidades nativas del Ecuador (DE LA TORRE et al., 2008).
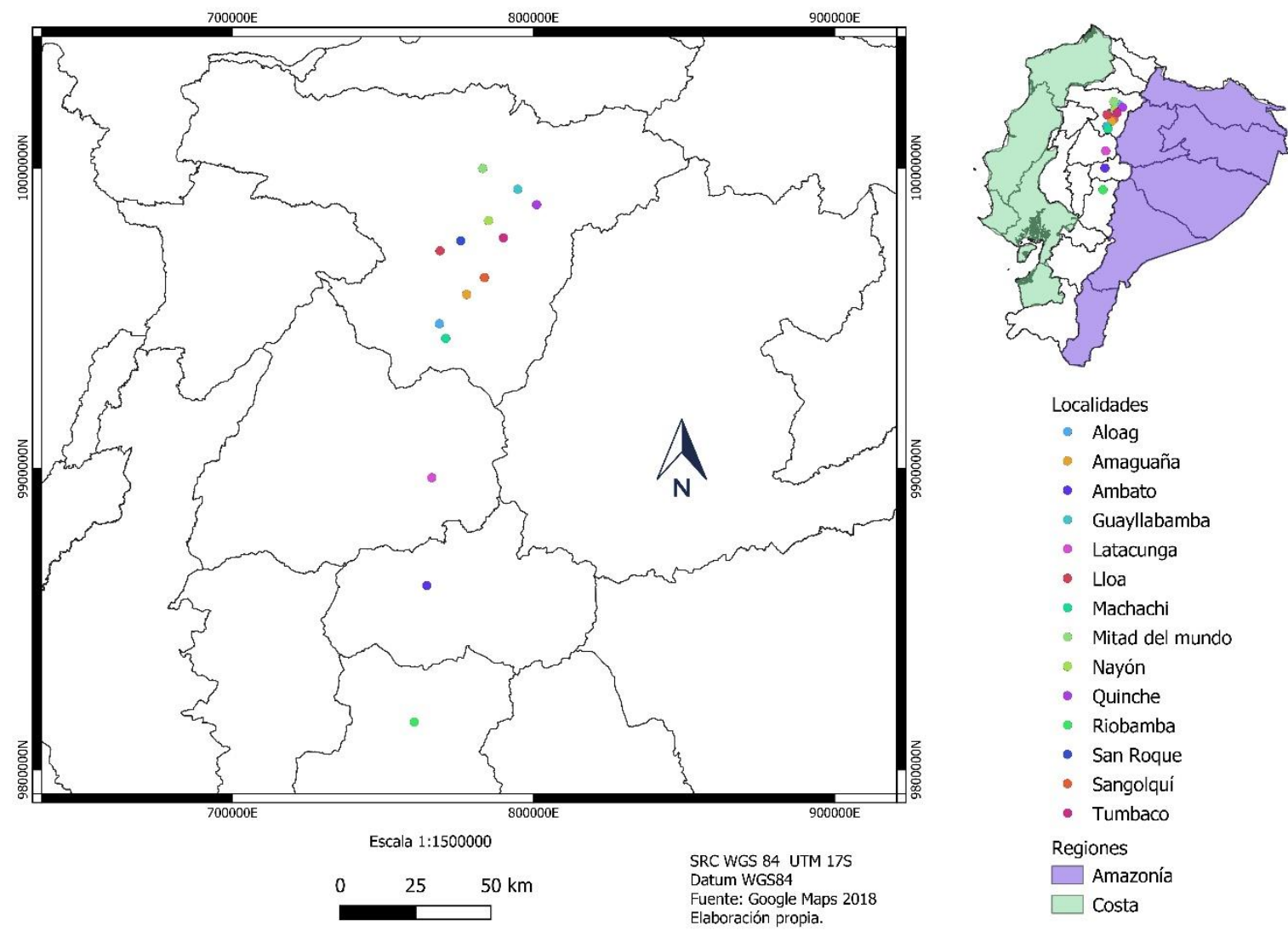

Figura 6: Mapa de las localidades mencionadas por las yerbateras.

\section{Similitud de conocimientos y plantas usadas entre mercados}

No encontramos diferencias significativas entre los conocimientos encontrados en cada uno de los mercados $(\mathrm{p}>0.05$ ) (Figura 7). Los mercados que visitamos al parecer utilizan generalmente las mismas especies de plantas, en nuestro NMDS no se observan grupos separables (Figura 8), la mayor cantidad de especies mencionadas por yerbateros están presentes en el mercado de San Roque. Estos dos hechos podrían deberse a que algunos de estos mercados son provistos por intermediarios con orígenes en común y que San Roque sea una fuente de abastecimiento para otros mercados.

\section{Relación de la edad y la experiencia de las yerbateras con sus conocimientos}

Existe una correlación negativa entre la edad y el número de plantas citadas por algunos yerbateros $(\mathrm{r}=-0.16)$, lo mismo sucede entre el tiempo trabajado en años y el número de plantas citadas $(\mathrm{r}=-0.18)$ (Figuras 9 y 10). Los conocimientos no aumentan de forma significativa con la edad y experiencia ( $>0.05)$ Otros estudios etnobotánicas suelen reportar relaciones positivas muy significativas (ESTRADA et al., 2001; ALBINO-GARCÍA et al., 2010). Como lo reportan Albino-García et al. (2010), en ocasiones, mayor edad u experiencia no siempre representa mayores conocimientos. Las causas podrían ser complejas y merecen ser investigadas. El envejecimiento de la memoria con el paso del tiempo podría ser una opción. Sugerimos que en otros estudios se deban tomar más datos en cuenta sobre estos aspectos, sumado a la autoidentificación étnica de cada uno de los yerbateros. Esto podría ser importante pues podrían existir diferencias significativas entre el conocimiento de grupos humanos que se dediquen a esta profesión y que hace falta descubrir. 


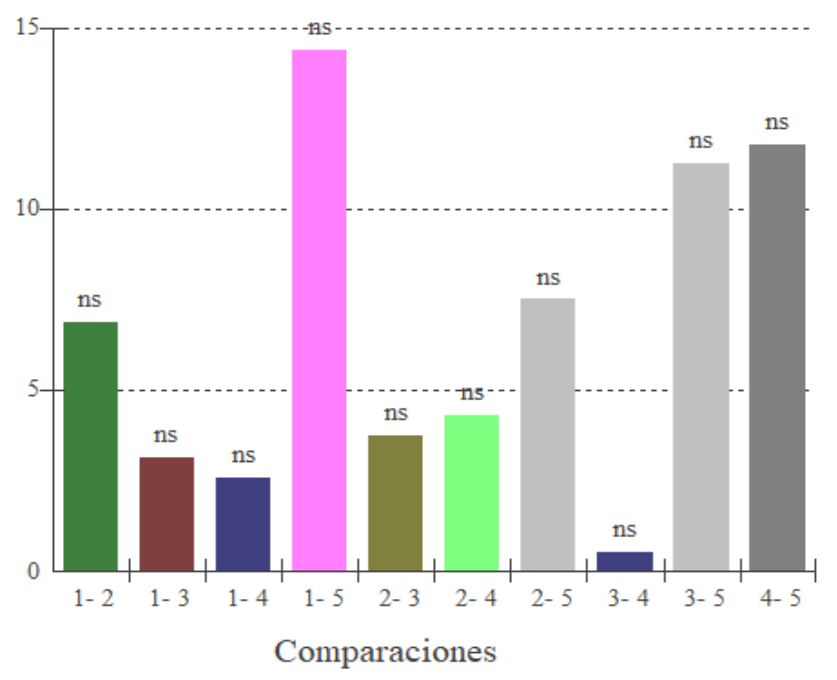

Figura 7: Test de Kruskall Wallis que compara el número de conocimientos mencionado por cada yerbatera entre los mercados visitados. Leyenda: 1=Mercado de Cotocollao, 2=Mercado Santa Clara, 3= Mercado San Franciso, 4= Mercado de San Roque, 5= Mercado Central, ns= no significativo. Estadìsticos: p=0.18, gl=4, $\mathrm{H}=6.25$.

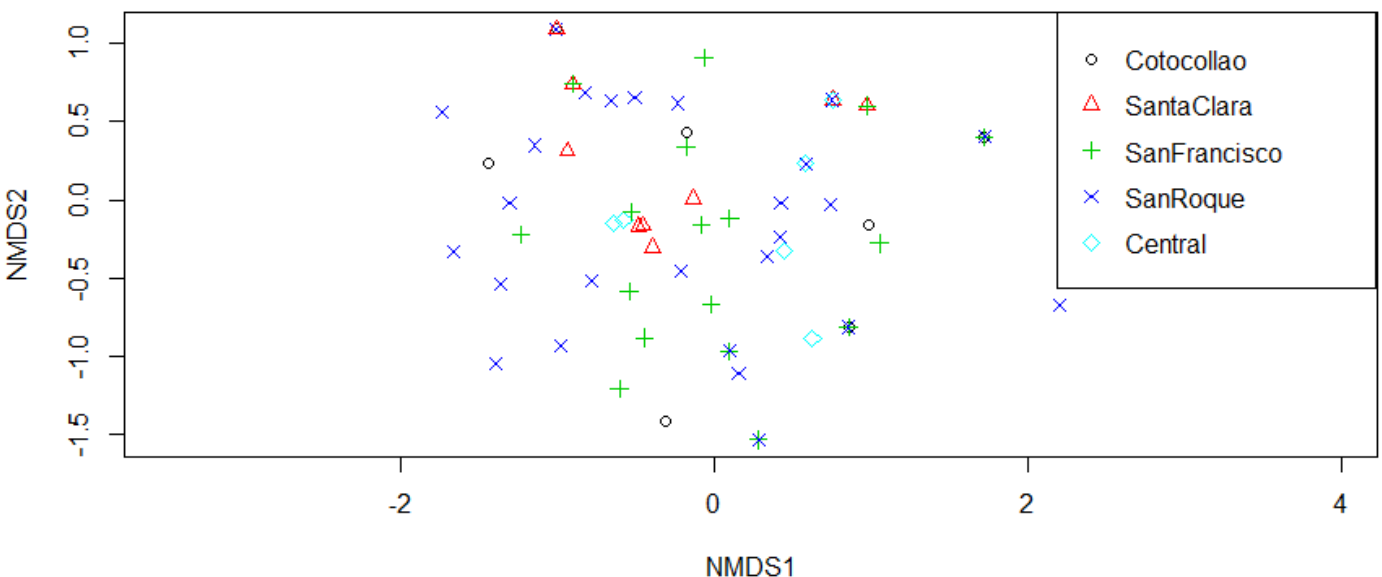

Figura 8: NMDS realizado en base a las especies presentes en cada uno de los mercados. El estrés de este ordenamiento se puede considera justo (Stress=0.1).

\section{Otros Usos}

Encontramos que el 52\% (47) de las especies de plantas en este trabajo son exclusivas de uso ritual mientras que un $48 \%$ (43) de las especies presentaron otros usos, principalmente dentro del ámbito Medicinal, es decir, 37\% (33) de las especies tenían usos rituales y medicinales a la vez (Anexo 1). Esto es superior a los resultados presentados por Maioli-Azevedo y Da Fonseca-Kruel (2007) en ferias libres de las zonas Norte y Sur de Rio de Janeiro en Brasil, donde encontraron 106 especies de plantas útiles, de las cuales apenas el 5,6\% tenían usos medicinales y rituales a la vez. 

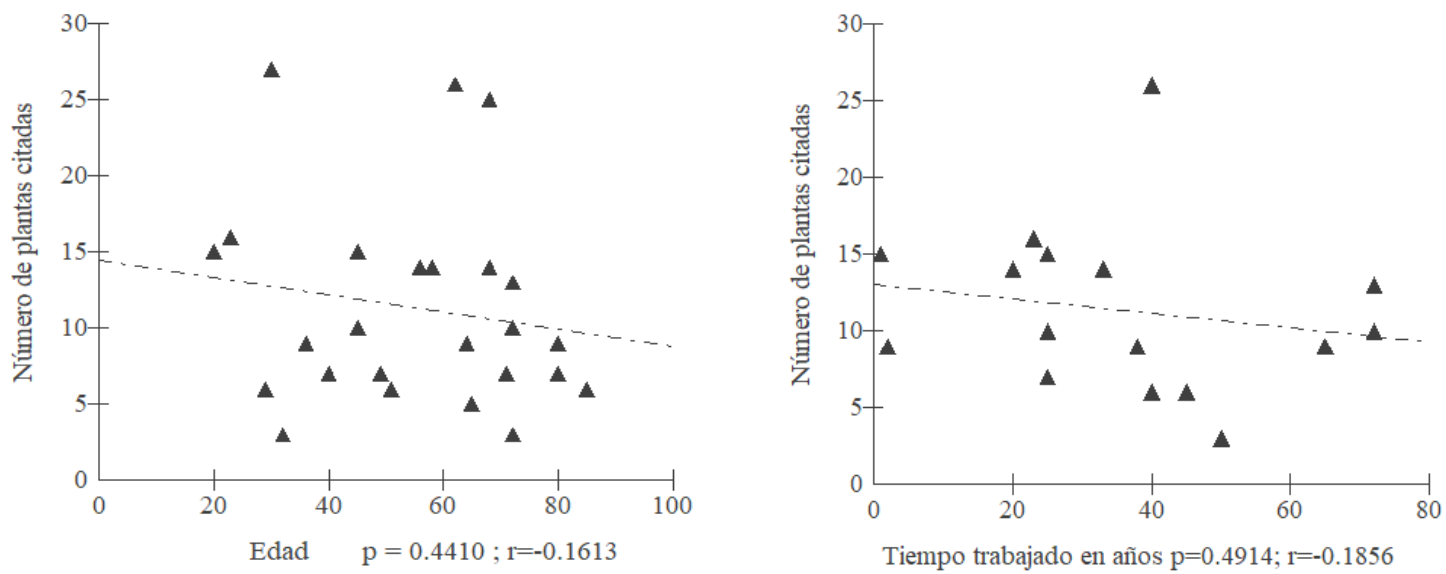

Figuras 9 y 10: A la izquierda: Correlación entre la edad y el número de plantas citadas por 25 yerbateras de las que se disponían datos ( $\mathrm{IC}=-0.62-0.34)$. A la derecha Correlación entre el tiempo trabajado en años y el número de plantas citadas para 16 yerbateras de las que se disponían datos $(\mathrm{IC}=-0.52-0.25)$.

Tabla 5: Otros usos de las plantas rituales comentados por las yerbateras de varios mercados de Quito.

\begin{tabular}{|c|c|c|}
\hline \# & Nombre Científico & Otros usos \\
\hline 1 & Ruta Graveolens & Abortiva, para limpiar el piso, presión alta, nervios, para cólicos menstruales \\
\hline 2 & Ambrosia arborescens & $\begin{array}{l}\text { Escobas para barrer, salpullidos, las semillas usadas para problemas nerviosos, dolores } \\
\text { musculares, repelente de pulgas. }\end{array}$ \\
\hline 3 & Urtica urens & Parálisis facial, anti estrés, circulación \\
\hline 4 & Baccharis latifolia & Baños, dolor de huesos, resfriados, dolor estomacal, Bebidas en té, dolor de rodillas \\
\hline 5 & Tanacetum parthenium & Escobas para barrer, purgante \\
\hline 6 & Cestrum affine & Vaporizaciones, problema de la piel, inflamaciones, nervios, gripe, problemas de caspa \\
\hline 7 & Eucalyptus globulus & Gripe \\
\hline 8 & Miconia crocea & Gripe \\
\hline 9 & Solanum nigrescens & Salpullidos, problemas de la piel, laringitis, amigdalitis, estreñimiento \\
\hline 10 & Matthiola incana & Problemas nerviosos, problemas del corazón, problemas del hígado y riñones. Estrés \\
\hline 11 & Tagetes erecta & Baños y creación de coronas florales \\
\hline 12 & Aloysia citriodora & Bebidas en té, relajante \\
\hline 13 & Salvia alvajaca & Dolor de huesos, escalofríos que sienten las mujeres embarazadas, condimento \\
\hline 14 & Buddleja bullata & Dolor de huesos, emplastos, vías urinarias \\
\hline 15 & Mentha pulegium & Bebidas en té \\
\hline 16 & Melissa officinalis & Problemas del corazón, bebidas en té \\
\hline 17 & Borago officinalis & Gripe y tos \\
\hline 18 & Dodonaea viscosa & Escobas para barrer \\
\hline 19 & Psidium guajava & Diabetes, presión alta \\
\hline 20 & Ilex guayusa & Energizante, problemas de fertilidad \\
\hline 21 & Artemisia absinthium & Colesterol elevado, hígado graso y para bajar de peso \\
\hline 22 & Peperomia inaequalifolia & Nervios, dolor de oído \\
\hline 23 & Aristolochia sp. & Cólicos menstruales \\
\hline 24 & Origanum majorana & Condimento, problemas de colerín \\
\hline 25 & Tagetes minuta & Condimento para sopa \\
\hline 26 & Myrcianthes hallii & Alimento \\
\hline 27 & Cynara scolymus & Alimento \\
\hline 28 & Clinopodium nubigenum & Problemas de gases y tos \\
\hline 29 & Verbena litoralis & Glucosa alta \\
\hline 30 & Ceratochloa pitensis & Purgante para perros \\
\hline 31 & Juglans neotropica & Dolores de cabeza \\
\hline 32 & Urtica leptophylla & Alimento \\
\hline 33 & Lavandula angustifolia & Problemas de acné \\
\hline 34 & Croton ferrugineus & Afecciones del páncreas, té \\
\hline 35 & Matricaria chamomilla & Cuidado de la dentadura \\
\hline 36 & Maytenus macrocarpa & Dolor de huesos \\
\hline 37 & Cymbopogon citratus & Bebidas en té \\
\hline 38 & Varronia sp. & Problemas en vías urinarias \\
\hline 39 & Ocimum basilicum & Condimento para sopa \\
\hline 40 & Otholobium mexicanum & Dolor estomacal \\
\hline 41 & Verbena brasiliensis & Problemas de glucosa alta \\
\hline 42 & Iresine sp. & Cólicos menstruales y problemas de Riñón \\
\hline 43 & Monnina phillyreoides & Condimento de cocina \\
\hline
\end{tabular}




\section{CONCLUSIONES}

Este trabajó logró documentar una gran variedad de creencias y múltiples formas de uso de plantas rituales utilizadas por las yerbateras de la ciudad de Quito. Se reportan posibles usos rituales nuevos para varias especies. Algunos de ellos están relacionados a creencias bastante interesantes y peculiares. Las plantas europeas denotan la fuerte influencia de la colonización española en Ecuador y procesos de transculturización en las yerbateras de Quito. Estas plantas estaban en la categoría de preocupación menor, mientras una gran mayoría de plantas nativas no estaban evaluadas y son poco conocidas para las ciencias biológicas.

El estado de conservación no solo involucra el estado de la integridad de las especies, también involucra los conocimientos y usos tradicionales que existen sobre ellas. Se desconoce si las plantas usadas por los yerbateros provienen del extractivismo, de manera que quedan abiertas interrogantes sobre el cultivo, biomasa, intensidad de uso, estado de conservación y biología de muchas especies.

La mayoría de los intermediarios que traen plantas hacia los mercados visitados, las traen de puntos específicos en la región Sierra, el resto se traen de las provincias de la Costa y Amazonía ecuatoriana. En general las plantas utilizadas en cada mercado y por cada yerbatera son las mismas. Una mayor edad y experiencia de las yerbateras no demostró que existiesen más conocimientos. Los índices revisados reflejan preferencias de uso por parte de las yerbateras. Una buena parte de las plantas encontradas poseían otros usos, especialmente medicinales, al parecer estas plantas son ritualísticas y medicinales a la vez. Este trabajo segregó ambas categorías para dar un mayor enfoque a lo que se puede considerar ritual y logró documentar con mayor detalle este aspecto.

\section{AGRADECIMIENTOS}

Queremos agradecer a todas las personas que colaboraron con nosotros para la elaboración de este trabajo con su consentimiento previo informado: del mercado de Cotocollao y sus alrededores: María Valencia, Blanca Marina Pallo, Olga Chiluisa (trabaja 45 años como yerbatera) y Ricardo Gualoto (trabaja 25 años como yerbatero); del mercado San Francisco y sus alrededores: Emperatriz García (trabaja 72 años como yerbatera), Blanca Ganchala (trabaja 40 años como yerbatera), Mercedes Correa , Miriam Yánez (Trabaja 72 años como yerbatera), Asunción Pillajo y María Narváez (trabaja 50 años como yerbatera); del Mercado de San Roque: Cristina Guanolema, Viviana Quishpe, Luz María (trabaja 65 años como yerbatero), Marcela Cocha (trabaja 33 años como yerbatero), Patricia Chicaiza (trabaja 25 años como yerbatero), Lesly Espinoza (trabaja 1 año como yerbatero) y Liliana Mora (trabaja 23 años como yerbatero); del Mercado Central: Rosa María Duque, Nancy Pintag, Mayra Tipan (trabaja 40 años como yerbatero) y Luis Vargas.; del Mercado Santa Clara: Jesús Azuaje (trabaja 2 años como yerbatero) y Alexandra Sambache (trabaja 25 años como yerbatero). A los Doctores Paúl Gamboa Trujillo, Carlos Cerón Martínez y Juan Pablo Carrera por el apoyo brindado. Y a todos aquellos anónimos que fueron muy amables con nosotros.

\section{REFERENCIAS BIBLIOGRÁFICAS}

ADAMS, J.; SIBBRITT, D.; YOUNG, A. F. Consultations with a naturopath or herbalist: the prevalence of use and profile of users amongst mid-aged women in Australia. Public Health., v. 121, n. 12, p. 954-7, 2007.

ALBINO-GARCÍA, C.; CERVANTES, H.; LÓPEZ, M.; RÍOS-CASANOVA, L.; LIRA, R. Patrones de diversidad y aspectos etnobotánicos de las plantas arvenses del valle de Tehuacán-Cuicatlán: el caso de San Rafael, municipio de Coxcatlán, Puebla. Revista Mexicana de Biodiversidad, v. 82, p. 1005-1019, 2011.

ÁLVAREZ, D.; MIRANDA, M. I. Oficios que cuidan la vida: partería y curación. ÍCONOS Rev Ciencias Soc., v. 18, n. 3, p. 18, 2014.

AZEVEDO, S. K. S.; SILVA, I. M. Comercialização de plantas medicinais e de uso religioso no município do Rio de Janeiro. Acta Botanica Brasilica, v. 20, n. 1, p. 185-194, 2006.

BARRERA, G.; KUKLINSKI, J. De los yerbateros con sus hierbas: creaciones no hegemónicas en la plaza Samper Mendoza. Tabula Rasa, v. 29, p. 277-294, 2018.

BECERRA, M. H.; ADELINA, M.; PALADINES, R.; MALDONADO, M. Efectos psicológicos en los rituales ancestrales en Saraguro-Ecuador. Int J Dev Educ Psychol INFAD Rev Psicol., v. 1, n. 1, p. 463, 2020.

CARAPIA, L.; VIDAL, F. Etnobotánica: el estudio de la relación de las plantas con el hombre. Inecol-Conacyt. 2013. Disponible en: <https://www.inecol.mx/inecol/index.php/es/2013-06-05-10-34-10/17-ciencia-hoy/373-etnobotanica-elestudio-de-la-relacion-de-las-plantas-con-el-hombre. > Acceso en: 28 Oct 2019.

CABANILLA, E. Impactos culturales del turismo comunitario en Ecuador sobre el rol del chamán y los ritos mágicoreligiosos. Estud y Perspect en Tur., v. 24, n. 2, p. 356-373, 2015.

CERÓN, C. Manual de Botánica: sistemática, etnobotánica y métodos de estudio en el Ecuador. 1ra ed. Quito: Editorial Universitaria, 2003. 
CONABIO. Spartium junceum. Método de Evaluación Rápida de Invasividad (MERI) para especies exóticas en México. 2016 Disponible en:

<https://www.gob.mx/cms/uploads/attachment/file/221059/Spartium_junceum_final.pdf. >Acceso en: 20 feb 2020.

CLAVIJO, J. C.; YANEZ, P. Plantas frecuentemente utilizadas en zonas rurales de la región amazónica, centro occidental de Ecuador. INNOVA Res J., v. 2, n. 6, p. 9-21, 2017.

DE LA TORRE, L.; NAVARRETE, H.; MURIEL, P.; MACÍA, M.; BALSLEV, H. Enciclopedia de las plantas útiles del Ecuador. 1ra ed. Quito: Pontificia Universidad Católica del Ecuador PUCE, 2008.

DÍAZ, M.; FARRERA, O.; ISIDRO, M. Estudio etnobotánico de los principales mercados de Tuxtla Gutierrez, Chiapas, México. LACODONIA, v. 5, n. 2, p. 21-42, 2011.

ESTRADA, E.; AGUIRRE, J, R.; SANCHEZ, L. Tecnología tradicional y conocimiento etnobotánico forestal en Santa Isabel Chalma, Amecameca, México. Revista de Geografía Agrícola, v. 32, p. 43-74, 2001.

FARIÑA, Á.; RUIZ-VELÁSQUEZ, L.; ROJAS, M.; PEÑUELA, J.; GONZÁLES, N. Etnobiología marina y aspectos pesqueros en seis comunidades costeras de la Península de Paria, Venezuela. Interciencia, v. 36, n. 4, p. 256-264. 2011.

FRIEDMAN, J., YANIV, Z.; DAFNI, A.; PALEWITCH, D. A preliminary classification of the healing potential of medicinal plants, based on a rational analysis of an ethnopharmacological field survey among Bedouins in the Negev Desert, Israel. Journal of Ethnopharmacology, v. 16, p. 275-287, 1986.

GARCÍA, J. R.; FIGUEROA, F. Cultura, interculturalidad, transculturalidad: elementos de y para un debate. Antropología sociológica, v. 9, p. 15-62, 2007.

HANAN, A.; MONDRAGÓN, J. Spartium junceum. Malezas de México. 2009 Disponible en: <http://www.conabio.gob.mx/malezasdemexico/fabaceae/spartium-junceum/fichas/ficha.htm>. Acceso en: 20 feb 2020.

HOFFMAN, B.; GALLAHER, T. Importance indices in ethnobotany. Ethnobot Research and Applications, v. 5, p. 201-218, 2007.

HOLLENSTEIN, P. ¿Están en riesgo los mercados y ferias municipales? Aprovisionamiento de alimentos, economías populares y la organización del espacio público urbano de Quito. 1ra ed. Quito: Friedrich-Ebert-Stiftung Ildis Ecuador, 2019.

JARAMILLO, M.; CASTRO, M.; RUIZ-ZAPATA, T.; LASTRES, M.; TORRECILLA, P.; LAPP, M.; HERNÁNDEZCHONG, L.; MUÑOZ, D. Estudio etnobotánico de plantas medicinales en la comunidad campesina de Pelelojo, Municipio Urdaneta, Estado Aragua, Venezuela. Ernstia., v. 24, n. 1, p. 85-110, 2014.

KVIST, L.; MORAE, M. Plantas psicoactivas. Botánica Económica los Andes Cent., v. 2, p. 19, 2006.

LEÓN-YÁNEZ, S.; VALENCIA, R.; PITMAN, N.; ENDARA, L.; ULLOA, C.; NAVARRETE, H. Libro rojo de las plantas endémicas del Ecuador. 2da ed. Quito: Publicaciones del Herbario QCA, Pontificia Universidad Católica del Ecuador, 2011.

LOPES, T. C.; COSTA, W.; DANTAS DE ALMEIDA, J. D. Erveiros (as) do Ver-o-Peso, em Belém do Pará: um estudo etnográfico. Revista África e Africanidades, n. 9, 2010.

MAIOLI-AZEVEDO, V.; DA FONSECA-KRUEL, V. S. Plantas medicinales y ritualísticas vendidas en ferias libres del Municipio de Rio de Janeiro, RJ, Brasil: Estudio de caso de las zonas Norte y Sur. Acta Bot Brasilica, v. 21, n. 2, p. 263 275, 2007.

MARÍN-CORBA, C.; CÁRDENAS-LÓPEZ, C.; SUÁREZ- SUÁREZ, S. Utilidad del valor de uso en etnobotánica. Estudio en el Departamento de Putumayo (Colombia). Caldasia, v. 27, p. 89-101, 2005.

MCDONALD, J. H. Handbook of Biological Statistics. 3rd ed. Baltimore: Sparky House Publishing, 2014. p. 190-180. Disponible en: <http://www.biostathandbook.com/linearregression.html> Acceso: en 24 feb 2020.

MINISTERIO DEL AMBIENTE DEL ECUADOR (MAE). Cuarto informe nacional para el convenio sobre la diversidad biológica. 1ra ed. Quito: MAE, 2010.

PHILLIPS, O.; GENTRY, A. H. The useful plants of Tambopata, Peru: I. Statistical hypotheses tests with a new quantitative technique. Economic Botany, v. 47, p. 15-32, 1993a.

PHILLIPS, O.; GENTRY, A. H. 1993. The useful plants of Tambopata, Peru: II. Additional hypothesis testing in quantitative ethnobotany. Economic Botany, v. 47, p. 33-43, 1993b.

QUINTANA, C. Plantas silvestres de los valles secos cercanos a Quito. 1ra Ed. Quito-Ecuador. Publicaciones del Herbario QCA, PUCE, 2013. 270 pp.

RAMOS, M.; ÁVILA, C.; MORALES, J. Etnobotánica y ecología de plantas utilizadas por tres curanderos contra la mordedura de serpiente en la región de Acayucan, Veracruz, México. Bol. Soc. Bot. Méx. v. 81, p. 89-100, 2007.

RIOS, M.; KOZIOL, M. J.; BORGTOFT PEDERSEN, H.; GRANDA, G. Plantas útiles del Ecuador: aplicaciones, retos y perspectivas. 1ra Ed. Quito: Corporación Sociedad para la Investigación y Monitoreo de la Biodiversidad Ecuatoriana (SIMBIOE), 2007.

RODRÍGUEZ, F.; ALVÍTEZ, E.; POLLACK, L.; HUAMÁN, E.; RIMARACHÍN, V.; MIRANDA, S.; PIZARRO, Y. Estado actual de la flora con pelos urticantes en la región la libertad, Perú. Sagasteguiana, v. 2, n. 2, p. 113-134, 2014

RODRÍGUEZ, Y.; VALDÉS, M.; HERNÁNDEZ, R.; SORIA, S. Guía metodológica para estudios etnobotánicos de especies forestales en comunidades amazónicas y afines. Revista Cubana de Ciencias Forestales, v. 7, n. 1, p. 98-110, 2018.

UICN. The UICN red list of threatened species. 2020. Disponible en: <https://www.iucnredlist.org/.> Acceso: en 24 feb 2020.

VALENCIA, R. PITMAN, N.; LEÓN-YÁNEZ, S.; JØRGENSEN P. M. Libro rojo de las plantas endémicas del Ecuador. 1ra Ed. Quito: Publicaciones del Herbario QCA. Pontificia Universidad Católica del Ecuador, 2000.

ZAMBRANO-INTRIAGO, L. F.; BUENAÑO-ALLAUCA, M. P.; MANCERA-RODRÍGUEZ, J. N.; JIMÉNEZROMERO, E. Estudio etnobotánico de plantas medicinales utilizadas por los habitantes del área rural de la Parroquia San Carlos, Quevedo, Ecuador. Rev Univ salud., v. 17, n. 1, p. 97-111, 2015.

ZEBADÚA, J. Cultura, identidades y transculturalidad. Apuntes sobre la construcción identitaria de las juventudes indígenas. Revista Limina R. Estudios sociales y humanísticos, v. 9, n. 1, 2011. 


\section{Anexo 1}

Tabla 4: Resumen de datos etnobotánicos.

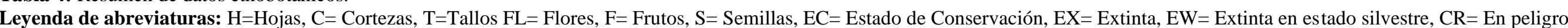

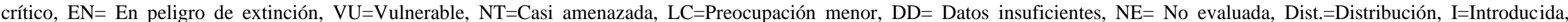

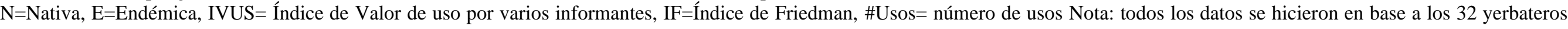
entrevistados.

\begin{tabular}{|c|c|c|c|c|c|c|c|c|c|c|}
\hline \# & Nombres comunes & Nombre Científico & Familia & Propósitos y Formas de Uso & $\begin{array}{l}\text { Parte } \\
\text { usada }\end{array}$ & Dist. & EC & \#Usos & IVUs & IF \\
\hline 1 & Ruda & Ruta Graveolens L. & Burseraceae & $\begin{array}{l}\text { Alejar malas energías o malos aires, curar el espanto, limpias, baños amargos, } \\
\text { cultivada en la entrada de las casas para alejar la envidia }\end{array}$ & $\mathrm{H}, \mathrm{T}, \mathrm{FL}$ & I & LC & 9 & 1.28 & 90.63 \\
\hline 2 & Marco & Ambrosia arborescens Mill. & Asteraceae & Alejar malas energías o malos aires, curar el espanto, baños amargos, limpias, & $\mathrm{H}, \mathrm{T}, \mathrm{FL}$ & $\mathrm{N}$ & $\mathrm{NE}$ & 8 & 1.00 & 68.75 \\
\hline 3 & Ortiga Negra & Urtica urens $\mathrm{L}$. & Urticaceae & $\begin{array}{l}\text { Alejar malas energías o malos aires, curar el espanto, quitar el estrés, baños } \\
\text { amargos, limpias, }\end{array}$ & $\mathrm{H}, \mathrm{T}, \mathrm{FL}$ & $\mathrm{I}$ & LC & 7 & 0.97 & 62.50 \\
\hline 4 & Chilca & Baccharis latifolia (Ruiz \& Pav.) Pers & Asteraceae & Alejar malas energías o malos aires, curar el espanto, baños amargos, limpias, & $\mathrm{H}, \mathrm{T}$ & $\mathrm{N}$ & LC & 9 & 0.91 & 59.38 \\
\hline 5 & Santa María & Tanacetum parthenium (L.) Sch.Bip & Asteraceae & Alejar malas energías o malos aires, curar el espanto, baños amargos, limpias, & $\mathrm{H}, \mathrm{T}, \mathrm{FL}$ & I & LC & 5 & 0.84 & 75.00 \\
\hline 6 & Sauco & Cestrum affine Kunth & Solanaceae & Alejar malas energías o malos aires, curar el espanto, baños amargos, limpias, & $\mathrm{H}, \mathrm{T}$ & $\mathrm{N}$ & $\mathrm{NE}$ & 9 & 0.78 & 53.13 \\
\hline 7 & Eucalipto & Eucalyptus globulus Labill. & Myrtaceae & Alejar malas energías o malos aires, curar el espanto, baños amargos, limpias & $\mathrm{H}, \mathrm{T}$ & I & LC & 4 & 0.63 & 46.88 \\
\hline 8 & Colca & Miconia crocea (Desr.) Naudin & Melastomataceae & Alejar malas energías o malos aires, curar el espanto, baños amargos, limpias & $\mathrm{H}, \mathrm{T}$ & $\mathrm{N}$ & $\mathrm{NE}$ & 4 & 0.41 & 34.38 \\
\hline 9 & Hierba Mora & $\begin{array}{l}\text { Solanum nigrescens. M. Martens \& } \\
\text { Galeotti }\end{array}$ & Solanaceae & Alejar malas energías o malos aires, baños energéticos, limpias & $\mathrm{H}, \mathrm{T}, \mathrm{FL}, \mathrm{F}$ & $\mathrm{N}$ & $\mathrm{NE}$ & 7 & 0.34 & 15.63 \\
\hline 10 & Alelí, Alegrías & Matthiola incana (L.) R.Br. & Brassicaceae & Alejar malas energías o malos aires, baños dulces & FL & I & $\mathrm{NE}$ & 7 & 0.34 & 12.50 \\
\hline 11 & Flor de Muerto, Ayarosa & Tagetes erecta $\mathrm{L}$. & Asteraceae & Alejar malas energías o malos aires, baños amargos, limpias, & FL & I & $\mathrm{NE}$ & 3 & 0.31 & 25.00 \\
\hline 12 & Capulí & Prunus serótina Ehrh. & Rosaceae & Alejar malas energías o malos aires, baños amargos, limpias & $\mathrm{H}, \mathrm{T}$ & $\mathrm{N}$ & LC & 2 & 0.19 & 18.75 \\
\hline 13 & Romero & Rosmarinus officinalis $\mathrm{L}$. & Lamiaceae & Baños dulces & $\mathrm{H}, \mathrm{T}$ & I & LC & 1 & 0.19 & 18.75 \\
\hline 14 & Tzímbalo & Solanum caripense Dunal & Solanaceae & Alejar malos aires de los niños, quitar el mal de ojo, collares & $\mathrm{F}$ & $\mathrm{N}$ & $\mathrm{NE}$ & 2 & 0.19 & 15.63 \\
\hline 15 & Guanto, Floripondio & $\begin{array}{l}\text { Brugmansia sanguínea (Ruiz \& Pav.) } \\
\text { D.Don }\end{array}$ & Solanaceae & Alejar malas energías o malos aires, curar el espanto, baños amargos, limpias, & FL & $\mathrm{N}$ & EW & 3 & 0.16 & 15.63 \\
\hline 16 & Chagrillo, Tigrecillo & Peperomia galioides Kunth & Piperaceae & Alejar malas energías o malos aires, baños & $\mathrm{H}, \mathrm{T}$ & $\mathrm{N}$ & $\mathrm{NE}$ & 2 & 0.16 & 18.75 \\
\hline 17 & Cedrón & Aloysia citriodora Palau & Verbenaceae & Alejar malas energías o malos aires, infusión & $\mathrm{H}, \mathrm{T}, \mathrm{FL}$ & $\mathrm{N}$ & $\mathrm{NE}$ & 3 & 0.16 & 6.25 \\
\hline 18 & Albaca dulce & Salvia alvajaca Oerst. & Lamiaceae & Baños dulces, limpias & $\mathrm{H}, \mathrm{T}$ & I & $\mathrm{NE}$ & 4 & 0.16 & 6.25 \\
\hline 19 & Quishuar, Matico & Buddleja bullata Kunth & Scrophulariaceae & Alejar malas energías o malos aires, baños amargos, limpias & $\mathrm{H}, \mathrm{T}, \mathrm{FL}$ & $\mathrm{N}$ & $\mathrm{NE}$ & 5 & 0.16 & 3.13 \\
\hline 20 & Ciprés & Cupressus lusitánica Mill. & Cupressaceae & Alejar malas energías o malos aires, baños amargos, limpias & $\mathrm{H}, \mathrm{T}$ & I & $\mathrm{LC}$ & 2 & 0.13 & 9.38 \\
\hline 21 & Laurel & $\begin{array}{l}\text { Morella pubescens (Humb. \& Bonpl. } \\
\text { Ex Willd.) Wilbur }\end{array}$ & Myricaceae & Alejar malas energías o malos aires, baños amargos, limpias, & $\mathrm{H}, \mathrm{T}$ & $\mathrm{N}$ & LC & 2 & 0.13 & 12.50 \\
\hline 22 & Menta & Mentha pulegium $\mathrm{L}$. & Lamiaceae & Alejar malas energías o malos aires, baños dulces, infusión & $\mathrm{H}, \mathrm{T}$ & I & LC & 3 & 0.13 & 3.13 \\
\hline 23 & Toronjil & Melissa officinalis $\mathrm{L}$. & Lamiaceae & Alejar malas energías o malos aires, infusión & $\mathrm{H}, \mathrm{T}$ & I & LC & 3 & 0.13 & 6.25 \\
\hline 24 & Escancel, Borraja & Borago officinalis L. & Boraginaceae & Alejar malas energías o malos aires, limpias & $\mathrm{H}, \mathrm{T}$ & I & $\mathrm{LC}$ & 3 & 0.13 & 6.25 \\
\hline 25 & Chamana & Dodonaea viscosa (L.) Jacq. & Sapindaceae & $\begin{array}{l}\text { Alejar malas energías o malos aires, baños amargos, baños de buena suerte, } \\
\text { limpias }\end{array}$ & $\mathrm{H}, \mathrm{T}, \mathrm{FL}, \mathrm{F}$ & $\mathrm{N}$ & LC & 4 & 0.13 & 9.38 \\
\hline 26 & Guayaba, Laurel de dulce & Psidium guajava $\mathrm{L}$. & Myrtaceae & Alejar malas energías o malos aires, baños amargos, baños dulces, limpias, & $\mathrm{H}, \mathrm{T}$ & $\mathrm{N}$ & LC & 5 & 0.13 & 12.50 \\
\hline 27 & Guayusa & Ilex guayusa Loes. & Aquifoliaceae & Alejar malas energías o malos aires, infusión, baños & $\mathrm{H}$ & $\mathrm{N}$ & LC & 3 & 0.13 & 6.25 \\
\hline 28 & Ajenjo & Artemisia absinthium L. & Asteraceae & Alejar malas energías o malos aires, limpias & $\mathrm{H}, \mathrm{T}$ & $\mathrm{I}$ & LC & 4 & 0.13 & 3.13 \\
\hline 29 & Cordoncillo & Piper barbatum Kunth & Piperaceae & Alejar malas energías o malos aires, baños amargos, limpias, & $\mathrm{H}, \mathrm{T}$ & $\mathrm{N}$ & $\mathrm{NE}$ & 2 & 0.13 & 12.50 \\
\hline 30 & Chagrillo, Congona & Peperomia inaequalifolia Ruiz \& Pav. & Piperaceae & Alejar malas energías o malos aires, baños dulces, limpias, & $\mathrm{H}, \mathrm{T}, \mathrm{FL}$ & $\mathrm{N}$ & $\mathrm{NE}$ & 4 & 0.13 & 9.38 \\
\hline
\end{tabular}




\begin{tabular}{|c|c|c|c|c|c|c|c|c|c|c|}
\hline \# & Nombres comunes & Nombre Científico & Familia & Propósitos y Formas de Uso & $\begin{array}{l}\text { Parte } \\
\text { usada }\end{array}$ & Dist. & EC & \#Usos & IVUs & IF \\
\hline 31 & Zaragoza & Aristolochia sp. L. & Aristolochiaceae & Baños contra la brujería & $\mathrm{T}$ & $\mathrm{N}$ & NE & 2 & 0.13 & 9.38 \\
\hline 32 & Mejorana, Domina todo & Origanum majorana L. & Lamiaceae & Baños dulces, baños de buena suerte & $\mathrm{H}, \mathrm{T}$ & $\mathrm{I}$ & $\mathrm{NE}$ & 4 & 0.13 & 6.25 \\
\hline 33 & Orégano de sal & Origanum vulgare $\mathrm{L}$. & Lamiaceae & Alejar malas energías o malos aires, limpias & $\mathrm{H}, \mathrm{T}$ & I & $\mathrm{LC}$ & 1 & 0.09 & 9.38 \\
\hline 34 & Chinchín & $\begin{array}{l}\text { Senna multiglandulosa (Jacq.) } \\
\text { HSIrwin \& Barneby }\end{array}$ & Fabaceae & Baños energéticos & $\mathrm{H}, \mathrm{T}$ & $\mathrm{N}$ & LC & 1 & 0.09 & 9.38 \\
\hline 35 & Asnayuyo, Tzintzo & Tagetes minuta $\mathrm{L}$. & Asteraceae & Alejar malas energías o malos aires, baños amargos limpias, & $\mathrm{H}, \mathrm{T}, \mathrm{FL}$ & $\mathrm{N}$ & $\mathrm{NE}$ & 3 & 0.09 & 9.38 \\
\hline 36 & Arrayán & Myrcianthes hallii (O.Berg) McVaugh & Myrtaceae & Alejar malas energías o malos aires, baños dulces, limpias, & $\mathrm{H}, \mathrm{T}$ & $\mathrm{N}$ & $\mathrm{NE}$ & 3 & 0.09 & 12.50 \\
\hline 37 & Chuquiragua & Chuquiraga jussieui J.F.Gmel. & Asteraceae & Alejar malas energías o malos aires, infusión & $\mathrm{H}, \mathrm{T}, \mathrm{FL}$ & $\mathrm{N}$ & $\mathrm{NE}$ & 1 & 0.09 & 9.38 \\
\hline 38 & Rosa & Rosa hybrida Vill. & Rosaceae & Alejar malas energías o malos aires, baños dulces & FL & I & $\mathrm{NE}$ & 2 & 0.09 & 9.38 \\
\hline 39 & Arquitecto & Lasiocephalus ovatus Schltdl & Asteraceae & Alejar malas energías o malos aires, baños & $\mathrm{H}, \mathrm{T}, \mathrm{FL}$ & $\mathrm{N}$ & $\mathrm{NE}$ & 1 & 0.09 & 9.38 \\
\hline 40 & Alcachofa & Cynara scolymus $\mathrm{L}$. & Asteraceae & Alejar malas energías o malos aires, infusión & $\mathrm{H}$ & I & $\mathrm{NE}$ & 2 & 0.09 & 6.25 \\
\hline 41 & Sunfo & $\begin{array}{l}\text { Clinopodium nubigenum (Kunth) } \\
\text { Kuntze }\end{array}$ & Lamiaceae & Alejar malas energías o malos aires, infusión & $\mathrm{H}, \mathrm{T}, \mathrm{FL}$ & $\mathrm{N}$ & $\mathrm{NE}$ & 3 & 0.09 & 3.13 \\
\hline 42 & Verbena, Verbena hembra & Verbena litoralis Kunth & Verbenaceae & Baños dulces & $\mathrm{H}, \mathrm{T}, \mathrm{FL}$ & $\mathrm{N}$ & NE & 2 & 0.09 & 6.25 \\
\hline 43 & Hierba de perro & Ceratochloa pitensis(Kunth) Holub & Poaceae & Alejar malas energías o malos aires, limpias & $\mathrm{H}, \mathrm{T}, \mathrm{FL}, \mathrm{S}$ & $\mathrm{N}$ & $\mathrm{NE}$ & 2 & 0.09 & 3.13 \\
\hline 44 & Tocte & Juglans neotropica Diels & Juglandaceae & Alejar malas energías o malos aires, baños amargos, limpias, & $\mathrm{H}$ & $\mathrm{N}$ & EN & 3 & 0.06 & 12.50 \\
\hline 45 & Ortiga blanca, Ortiga suave & Urtica leptophylla Kunth & Urticaceae & Alejar malas energías o malos aires, curar el espanto, baños amargos, limpias, & $\mathrm{H}, \mathrm{T}, \mathrm{FL}$ & $\mathrm{N}$ & LC & 4 & 0.06 & 3.13 \\
\hline 46 & Lavanda & Lavandula angustifolia Mill. & Lamiaceae & Alejar malas energías o malos aires, baños dulces, limpias, & $\mathrm{H}, \mathrm{T}, \mathrm{FL}$ & I & LC & 3 & 0.06 & 12.50 \\
\hline 47 & Mosquera & Croton ferrugineus Kunth & Euphorbiaceae & Alejar malas energías o malos aires, baños amargos, limpias, & $\mathrm{H}, \mathrm{T}, \mathrm{FL}$ & I & $\mathrm{LC}$ & 4 & 0.06 & 3.13 \\
\hline 48 & Manzanilla & Matricaria chamomilla $\mathrm{L}$. & Asteraceae & Baños dulces & $\mathrm{H}, \mathrm{T}, \mathrm{FL}$ & I & LC & 2 & 0.06 & 9.38 \\
\hline 49 & Pino & $\begin{array}{l}\text { Pinus patula Schiede ex Schltdl. \& } \\
\text { Cham. }\end{array}$ & Pinaceae & Baños amargos & $\mathrm{H}, \mathrm{T}$ & I & LC & 1 & 0.06 & 9.38 \\
\hline 50 & Achiote & Bixa Orellana L. & Bixaceae & Alejar malas energías o malos aires, baños energéticos & $\mathrm{H}$ & $\mathrm{N}$ & LC & 2 & 0.06 & 6.25 \\
\hline 51 & Chuchuhuazo & $\begin{array}{l}\text { Maytenus macrocarpa (Ruiz y Pav.) } \\
\text { Briq. }\end{array}$ & Celastraceae & Alejar malas energías o malos aires, baños contra la brujería & $\mathrm{C}$ & $\mathrm{N}$ & LC & 3 & 0.06 & 3.13 \\
\hline 52 & Eneldo & Foeniculum vulgare Mill. & Apiaceae & Baños dulces, limpias y & $\mathrm{H}, \mathrm{T}$ & I & LC & 1 & 0.06 & 6.25 \\
\hline 53 & Tomillo, Orégano dulce & Thymus vulgaris $\mathrm{L}$. & Lamiaceae & Alejar malos aires de los niños, limpias & $\mathrm{H}, \mathrm{T}$ & $\mathrm{I}$ & $\mathrm{LC}$ & 1 & 0.06 & 6.25 \\
\hline 54 & Hierba luisa & Cymbopogon citratus (DC.) Stapf & Poaceae & Baños energéticos & $\mathrm{H}$ & $\mathrm{I}$ & $\mathrm{NE}$ & 2 & 0.06 & 3.13 \\
\hline 55 & Abre caminos & kohleria sp. REGEL & Gesneriaceae & Baños de buena suerte & $\mathrm{H}, \mathrm{T}$ & $\mathrm{N}$ & $\mathrm{NE}$ & 1 & 0.06 & 6.25 \\
\hline 56 & Matico & Varronia sp. P. Browne & Boraginaceae & Alejar malas energías o malos aires, baños amargos, limpias & $\mathrm{H}, \mathrm{T}$ & $\mathrm{N}$ & $\mathrm{NE}$ & 3 & 0.06 & 3.13 \\
\hline 57 & $\begin{array}{l}\text { Arrastradora, Sígueme sígueme, llama } \\
\text { cliente }\end{array}$ & Selaginella diffusa Spring & Selaginellaceae & Atraer el amor y amarres amorosos, baños dulces, baños de buena suerte & $\mathrm{H}, \mathrm{T}$ & $\mathrm{N}$ & $\mathrm{NE}$ & 3 & 0.06 & 6.25 \\
\hline 58 & Albaca & Ocimum basilicum $\mathrm{L}$. & Lamiaceae & Alejar malas energías o malos aires, infusión & $\mathrm{H}, \mathrm{T}$ & I & NE & 2 & 0.06 & 3.13 \\
\hline 59 & $\begin{array}{l}\text { Zorroyuyo, Chumbiso, Chinchín, } \\
\text { Hierba de Chivo }\end{array}$ & Tagetes zypaquirensis Bonpl. & Asteraceae & Alejar malas energías o malos aires, baños & $\mathrm{H}, \mathrm{T}$ & $\mathrm{N}$ & $\mathrm{NE}$ & 2 & 0.06 & 9.38 \\
\hline 60 & Trinitaria & $\begin{array}{l}\text { Otholobium mexicanum (L. f.) JW } \\
\text { Grimes }\end{array}$ & Fabaceae & Alejar malas energías o malos aires, limpias & $\mathrm{H}, \mathrm{T}$ & $\mathrm{N}$ & $\mathrm{NE}$ & 2 & 0.06 & 3.13 \\
\hline 61 & Verbena, Verbena macho & Verbena brasiliensis Vell. & Verbenaceae & Baños dulces & $\mathrm{H}, \mathrm{T}, \mathrm{FL}$ & $\mathrm{N}$ & $\mathrm{NE}$ & 2 & 0.06 & 3.13 \\
\hline 62 & Escancel & Iresine sp. P. Browne & Amaranthaceae & Alejar malas energías o malos aires, baños & $\mathrm{H}, \mathrm{T}$ & $\mathrm{N}$ & $\mathrm{NE}$ & 4 & 0.06 & 3.13 \\
\hline 63 & Olivo & Olea europea L. & Oleaceae & Baños de buena suerte & $\mathrm{H}, \mathrm{T}$ & $\mathrm{I}$ & DD & 1 & 0.03 & 3.13 \\
\hline 64 & Floripondio & Brugmansia arbórea (L.) Steud. & Solanaceae & Alejar malas energías o malos aires, baños & FL & $\mathrm{N}$ & EW & 2 & 0.03 & 3.13 \\
\hline 65 & Chirimoya & Annona cherimola Mill. & Annonaceae & Alejar malas energías o malos aires, baños amargos, limpias & $\mathrm{H}, \mathrm{T}$ & $\mathrm{N}$ & LC & 2 & 0.03 & 3.13 \\
\hline 66 & Aguacate & Persea americana Mill. & Lauraceae & Alejar malas energías o malos aires, baños dulces, limpias & $\mathrm{H}, \mathrm{T}$ & $\mathrm{N}$ & LC & 2 & 0.03 & 6.25 \\
\hline 67 & Guaba & Inga insignis Kunth & Fabaceae & Alejar malas energías o malos aires, baños amargos, limpias & $\mathrm{H}$ & $\mathrm{N}$ & $\mathrm{LC}$ & 2 & 0.03 & 3.13 \\
\hline 68 & Eucalipto aromático & Corymbia citriodora (Hook.) KDHill & Myrtaceae & Alejar malas energías o malos aires, baños dulces, limpias & $\mathrm{H}, \mathrm{T}$ & I & LC & 2 & 0.03 & 6.25 \\
\hline
\end{tabular}




\begin{tabular}{|c|c|c|c|c|c|c|c|c|c|c|}
\hline \# & Nombres comunes & Nombre Científico & Familia & Propósitos y Formas de Uso & $\begin{array}{l}\text { Parte } \\
\text { usada }\end{array}$ & Dist. & EC & \#Usos & IVUs & IF \\
\hline & & \& LASJohnson & & & & & & & & \\
\hline 69 & Palo Santo & $\begin{array}{l}\text { Bursera graveolens (Kunth) Triana y } \\
\text { Planch. }\end{array}$ & Burseraceae & Alejar malas energías o malos aires, incienso & $\mathrm{T}$ & $\mathrm{N}$ & LC & 1 & 0.03 & 3.13 \\
\hline 70 & Retama & Spartium junceum $\mathrm{L}$. & Fabaceae & Alejar malas energías o malos aires, limpias & $\mathrm{H}, \mathrm{T}, \mathrm{FL}$ & I & LC & 1 & 0.03 & 3.13 \\
\hline 71 & Cholán, Purga & Tecoma stans (L.) Juss. ex Kunth & Bignoniaceae & Alejar malas energías o malos aires, baños amargos & $\mathrm{H}, \mathrm{T}$ & $\mathrm{N}$ & LC & 2 & 0.03 & 3.13 \\
\hline 72 & Chamico & Datura stramonium $\mathrm{L}$. & Solanaceae & Baños, infusión & FL & $\mathrm{N}$ & $\mathrm{NE}$ & 1 & 0.03 & 3.13 \\
\hline 73 & Níspero & Eryobotria japónica (Thunb.) Lindl & Rosaceae & Alejar malas energías o malos aires, baños dulces, limpias & $\mathrm{H}$ & I & $\mathrm{NE}$ & 2 & 0.03 & 3.13 \\
\hline 74 & Tabaco & Nicotiana tabacum $\mathrm{L}$. & Solanaceae & Alejar malas energías o malos aires, baños, limpias & $\mathrm{H}$ & I & $\mathrm{NE}$ & 1 & 0.03 & 3.13 \\
\hline 75 & Amor de hombre, Hoja de plata & Tradescantia zebrina Boose & Commelinaceae & Alejar malas energías o malos aires ,limpias & $\mathrm{H}, \mathrm{T}$ & $\mathrm{N}$ & $\mathrm{NE}$ & 1 & 0.03 & 3.13 \\
\hline 76 & Ayahuasca & $\begin{array}{l}\text { Banisteriopsis caapi (Spruce ex } \\
\text { Griseb.) Morton }\end{array}$ & Malpighiaceae & Alejar malas energías o malos aires ,infusión & $\mathrm{T}$ & $\mathrm{N}$ & $\mathrm{NE}$ & 1 & 0.03 & 3.13 \\
\hline 77 & Amanza guapos & Justicia sp. L. & Acanthaceae & $\begin{array}{l}\text { Dominar a una persona con mal genio para enamorarla, buscando de este modo } \\
\text { obtener la obediencia de la pareja de una mujer, baños }\end{array}$ & $\mathrm{H}, \mathrm{T}$ & $\mathrm{N}$ & $\mathrm{NE}$ & 1 & 0.03 & 3.13 \\
\hline 78 & Querendona & Begonia sp. L. & Begoniaceae & Atraer el amor y amarres amorosos, atraer clientela a los negocios, baños & $\mathrm{H}, \mathrm{T}$ & $\mathrm{N}$ & $\mathrm{NE}$ & 2 & 0.03 & 3.13 \\
\hline 79 & Destrancadera & $\begin{array}{l}\text { Monochaetum lineatum (D. Don) } \\
\text { Naudin }\end{array}$ & Melastomataceae & Atraer clientela a los negocios, baños & $\mathrm{H}, \mathrm{T}$ & $\mathrm{N}$ & NE & 1 & 0.03 & 3.13 \\
\hline 80 & Clavel & Dianthus caryophyllus $\mathrm{L}$. & Caryophyllaceae & Baños dulces & $\mathrm{H}, \mathrm{T}, \mathrm{FL}$ & I & $\mathrm{NE}$ & 1 & 0.03 & 3.13 \\
\hline 81 & Sábila & Aloe vera (L.) Burm.f. & Xanthorrhoeaceae & $\begin{array}{l}\text { Alejar malas energías o malos aires, alejar la envidia, se cultiva en las entradas } \\
\text { de las casas o es colgada en su entrada. }\end{array}$ & $\mathrm{H}$ & I & $\mathrm{NE}$ & 1 & 0.03 & 3.13 \\
\hline 82 & Jengibre & Zingiber officinale Roscoe & Zingiberaceae & Baños energéticos, baños contra la brujería & $\mathrm{T}$ & I & $\mathrm{NE}$ & 2 & 0.03 & 3.13 \\
\hline 83 & Canela & Cinnaтотит verum J.Presl & Lauraceae & Baños energéticos & $\mathrm{C}$ & I & $\mathrm{NE}$ & 1 & 0.03 & 3.13 \\
\hline 84 & Hierba del ángel & Desmodium molliculum (Kunth) DC & Fabaceae & Alejar malas energías o malos aires, limpias & $\mathrm{H}, \mathrm{T}$ & $\mathrm{N}$ & $\mathrm{NE}$ & 1 & 0.03 & 3.13 \\
\hline 85 & Iguilin & $\begin{array}{l}\text { Monnina phillyreoides (Bonpl.) B. } \\
\text { Eriksen }\end{array}$ & Polygalaceae & Alejar malas energías o malos aires, limpias & $\mathrm{H}, \mathrm{T}$, & $\mathrm{N}$ & $\mathrm{NE}$ & 2 & 0.03 & 3.13 \\
\hline 86 & Malva rosa & $\begin{array}{l}\text { Pelargonium citrosum Voigt ex } \\
\text { Breiter }\end{array}$ & Geraniaceae & Alejar malas energías o malos aires, limpias & $\mathrm{H}, \mathrm{T}$ & I & $\mathrm{NE}$ & 1 & 0.03 & 3.13 \\
\hline 87 & Piquiyuyo & $\begin{array}{l}\text { Margyricarpus pinnatus (Lam.) } \\
\text { Kuntze }\end{array}$ & Rosaceae & Alejar malas energías o malos aires, baños amargos, limpias & $\mathrm{H}, \mathrm{T}$ & $\mathrm{N}$ & $\mathrm{NE}$ & 2 & 0.03 & 3.13 \\
\hline 88 & Vara de la Justicia & Pariana radiciflora Sagot ex Döll & Poaceae & Alejar malas energías o malos aires, limpias & $\mathrm{H}$ & $\mathrm{N}$ & $\mathrm{NE}$ & 1 & 0.03 & 3.13 \\
\hline 89 & Sábila macho & Aloe maculata All. & Xanthorrhoeaceae & $\begin{array}{l}\text { Alejar malas energías o malos aires, alejar la envidia, la planta se cultiva en las } \\
\text { entradas de las casas o es colgada en la entrada }\end{array}$ & $\mathrm{H}$ & I & $\mathrm{NE}$ & 4 & 0.03 & 3.13 \\
\hline 90 & Vara de la Justicia & Phytelephas aequatorialis Spruce & Arecaceae & Atraer amor y amarres amorosos, limpias & $\mathrm{H}$ & E & NT & 1 & 0.03 & 3.13 \\
\hline
\end{tabular}




\section{RONDA 1 \\ Anexo 2}

ENTREVISTA SOBRE PLANTAS RITUALES UTILIZADAS POR YERBATERAS DE VARIOS MERCADOS DE QUITO-ECUADOR Y SU RELACIÓN CON DIVERSIDAS CREENCIAS. APORTES SOBRE SU ESTADO DE CONSERVACIÓN

Fecha: Lugar:

Nombre del informante: Edad: Responsable:

¿De dónde obtiene usted las plantas que comercializa, sabe si son cultivadas o tomadas de la naturaleza?

\begin{tabular}{|c|c|c|c|c|c|}
\hline $\begin{array}{l}\text { Parte 1: ¿Conoce usted } \\
\text { alguna planta utilizada para } \\
\text { rituales, algún mito o creencia } \\
\text { como curar el mal de ojo, } \\
\text { Alejar malas energías o } \\
\text { espíritus, ¿Cuáles? }\end{array}$ & $\begin{array}{l}\text { Identificación } \\
\underline{\text { taxonómica }}\end{array}$ & $\begin{array}{l}\text { ¿Con qué } \\
\text { propósito se } \\
\text { usa cada } \\
\text { planta? }\end{array}$ & $\begin{array}{l}\text { ¿Qué } \\
\text { parte de } \\
\text { la planta } \\
\text { se usa? }\end{array}$ & $\begin{array}{l}\text { ¿Cómo } \\
\text { se usa la } \\
\text { planta? }\end{array}$ & $\begin{array}{l}\text { ¿Algún Otro uso } \\
\text { aparte de ser } \\
\text { ritual? } \\
\text { Alimenticio, } \\
\text { medicinal, etc.? }\end{array}$ \\
\hline & & & & & \\
\hline & & & & & \\
\hline & & & & & \\
\hline & & & & & \\
\hline $\begin{array}{l}\text { Parte 2: ¿Conoces alguna de } \\
\text { estas plantas? (Preguntarle al } \\
\text { entrevistado por plantas que } \\
\text { mencionaron otros } \\
\text { entrevistados, y por plantas } \\
\text { que conocemos para estos } \\
\text { propósitos) }\end{array}$ & & & & & \\
\hline $\begin{array}{l}\text { Nombres Comunes de las } \\
\text { plantas }\end{array}$ & & & & & \\
\hline & & & & & \\
\hline & & & & & \\
\hline
\end{tabular}

Otros datos provistos por el informante: 